\title{
Expression of fatty acid synthase genes and their role in development and arboviral infection of Aedes aegypti
}

\author{
Nunya Chotiwan ${ }^{1}$, Carlos A. Brito-Sierra ${ }^{2,3}$, Gabriella Ramirez $^{1}$, Elena Lian ${ }^{1}$, Catherine A. \\ Hill $^{2,3}$ and Rushika Perera ${ }^{1, *}$
}

${ }^{1}$ Department of Microbiology, Immunology and Pathology, Colorado State University, Fort Collins, Colorado, USA

${ }^{2}$ Department of Entomology, Purdue University, West Lafayette, Illinois, USA

${ }^{3}$ Purdue Institute of Inflammation, Immunology and Infectious Disease, Purdue University, West Lafayette, Indiana, United States

* Correspondance: rushika.perera@colostate.edu

Email-address:

NC: nunya.chotiwan@gmail.com

CAB: cabritos@unal.edu.co

GR: Gabriela.Ramirez@ colostate.edu

EL: elian@ rams.colostate.edu

CAH: hillca@purdue.edu

RP: rushika.perera@colostate.edu 


\section{Abstract}

Background: Fatty acids are the building blocks of complex lipids essential for living organisms. In mosquitoes, fatty acids are involved in cell membrane production, energy conservation and expenditure, innate immunity, development, and reproduction. Fatty acids are synthesized by a multifunctional enzyme complex called fatty acid synthase (FAS). Several paralogues of FAS were found in the Aedes aegypti (Ae. aegypti) mosquito. However, the molecular characteristics and the expression of some of these paralogues have not been investigated.

Methods: Genome assemblies of Ae. aegypti were analyzed and orthologues of human FAS were identified. Phylogenetic analysis and in silico molecular characterization were performed to identify the functional domains of the Ae. aegypti FAS (AaFAS). Quantitative analysis and lossof-function experiments were performed to determine the significance of different $A a \mathrm{FAS}$ transcripts in various stages of development, expression following different diets and the impact of $A a \mathrm{FAS}$ on dengue virus, serotype 2 (DENV2) infection and transmission.

Results: We identified seven putative FAS genes in the Ae. aegypti genome assembly, based on nucleotide similarity to the FAS proteins (tBLASTn) of humans, other mosquitoes and invertebrates. Bioinformatics and molecular analyses suggested that only five of the FAS genes produce mRNA and therefore represent complete gene models. Expression levels of AaFAS varied among developmental stages and between male and female Ae. aegypti. Quantitative analyses revealed that expression of $A a \mathrm{FAS} 1$, the putative orthologue of the human FAS, was highest in adult females. Transient knockdown (KD) of AaFAS1 did not induce a complete 
compensation by other AaFAS genes but limited DENV2 infection of Aag2 cells in culture and the midgut of the mosquito.

Conclusion: $A a \mathrm{FAS} 1$ is the predominant $A a \mathrm{FAS}$ in the adult mosquitoes. It has the highest amino acid similarity to human FAS and contains all enzymatic domains typical of human FAS. AaFAS1 also facilitated DENV2 replication in both cell culture and in mosquito midguts. Our data suggest that AaFAS1 may play a role in transmission of dengue viruses, and could represent a target for intervention strategies.

Keywords: Aedes aegypti, Aag2 cells, fatty acid synthase, FAS, lipid, lipid metabolism, dengue virus, AaegL5 genome assembly 


\section{Background}

Fatty acid synthase (FAS) is a multifunctional enzyme catalyzing more than 40 steps in the $d e$ novo fatty acid biosynthesis pathway (1) (2). It contains seven catalytic and three non-catalytic domains which condense, reduce and dehydrate the three-carbon substrate, malonyl-CoA, into 16 to 18-carbon fatty acids. These fatty acids are essential building blocks of complex lipids, such as phosphoglycerolipids, glycerolipids and sphingolipids, which are components of cellular membranes and storage lipids, and function as signaling molecules, respectively.

In mosquitoes, fatty acids also play roles in innate immunity, reproduction, development, and flight (3-5). Fatty acids can be acquired or synthesized in both larvae and adult stages. Neonate larvae acquire lipids through the maternal deposition in eggs (6-8) and through consumption of aquatic diets such as diatoms and algae, which are the primary source of polyunsaturated fatty acids (9). Fatty acids from larval stages can be transferred to the adult stage and some can be deposited in eggs of the first gonotrophic cycle (10). Adult mosquitoes possess enzymes for de novo synthesis and modification of fatty acids from both sugar (carbohydrateenriched) and blood (protein-enriched) meals $(7,9,11)$. In the female, fatty acid synthesis is important for metabolism and production of eggs. Transient knockdown (KD) of acetyl-CoA carboxylase (ACC) and fatty acid synthase (FAS), two key enzymes in the de novo fatty acid biosynthesis pathway, led to significantly lower egg production in the first gonotrophic cycle (12). In addition, eggs produced by ACC-KD mosquitoes lacked eggshells and were nonviable (12).

Apart from its importance to mosquito biology, studies suggest FAS also plays a supportive role for several arboviral infections in both mammalian and mosquito cells (13-16). It is known that several RNA viruses induce expansion and rearrangement of host cell membranes 
to support viral genome replication and assembly (17-19). Studies have shown that FAS facilitates the production of dengue virus serotype 2 (DENV2) infection in both human and mosquito cells, potentially by providing the building blocks for this membrane expansion event $(13,15)$. Lastly, studies also reported the elevation of fatty acid abundance in C6/36 (Aedes albopictus) cells, and in the Aedes aegypti (Ae. aegypti) mosquito midgut during DENV2 infection $(15,20)$. These findings suggest that fatty acids are essential for the physiological function of mosquitoes, and support DENV2 infection of the mosquito.

Currently, understanding of FAS in mosquitoes and its role in pathogen transmission by the mosquito vector is limited. Here, we describe the molecular and functional characterization of the FAS gene family from Ae. aegypti (AaFAS). We identified seven putative AaFAS genes (AaFAS 1-6 and AaFAS-like) in the AaegL5 genome assembly, characterized the expression of these genes during mosquito development and following consumption of different diets. AaFAS1 had the highest amino acid similarity to human FAS and was the predominant transcript. We investigated the role of $A a \mathrm{FAS} 1$ in DENV2 infection in mosquito cells and live mosquitoes using gene KD. We observed a significant reduction of DENV2 replication following AaFAS1$\mathrm{KD}$ in Ae. aegypti cells and a transient reduction of infection in Ae. aegypti midguts at early time points post-infectious blood meal. These results provide insights to the molecular characteristic of $A a \mathrm{FASs}$ and their role during Ae. aegypti development, food source acquisition and arbovirus infection. 


\section{Methods}

\section{Alignments, conserved motifs and phylogenetic tree}

Putative AaFAS sequences from the AaegL5 genome assembly were blasted against the AaegL3 genome assembly retrieved from VectorBase using tBLASTn (21-23). FAS sequences of Anopheles gambiae, Drosophila melanogaster, Apis mellifera, Homo sapiens, Mus musculus and Saccharomyces cerevisiae were aligned with putative AaFAS sequences using ClustalW (24). mRNA sequences were retrieved from NCBI and manually curated to confirm the intron/exon boundaries. Conserved FAS motifs were identified by global alignment of vertebrate, invertebrate and yeast proteins using the Clustal Omega and Jalview 2.11.1.5 (accession numbers are shown in Table S1) (25) and conserved amino acids associated with catalytic domains of functional FAS were identified by comparison to sequences reported in published studies (26). Individual amino acid alignments were also performed between FAS-AaegL5 and FAS-AaegL3 using ClustalW to identify improvements in AaegL5 models.

A Bayesian inference of phylogeny was performed using the amino acid sequence of FAS from Ae. aegypti, Anopheles gambiae, Drosophila melanogaster, Apis mellifera, Mus musculus and Homo sapiens. Yeast Kexin was used as an outgroup. A sequence alignment with ClustalW was performed prior to tree construction in phylogeny.fr. The substitution model used for the Bayesian inference was Blosum62 and the Markov Chain Monte Carlo parameters included 100,000 generations with sampling every 10 generations, discarding the first 250 trees. The resulting tree was annotated and curated in iTOL. 


\section{Annotation of protein domains in Ae. aegypti FAS genes}

AaFAS amino acid sequences were aligned against the human FAS (NP_004095.4, NCBI) using Clustal Omega (27) to identify the seven catalytic and three noncatalytic domains associated with mammalian FAS. The alignment results were viewed using MView tool (28). Motifs in the human FAS were identified based on Pfam 31.0 (29) and conserved domains in AaFAS genes were identified by comparative analyses.

\section{Mosquito rearing}

Larvae and adults of Aedes aegypti strain Chetumal, originally collected from Yucatan Peninsula in Mexico, were reared on fish food and on $10 \%$ sucrose solution, respectively and adults maintained under constant conditions of $28^{\circ} \mathrm{C}, 80 \%$ relative humidity (30).

\section{Blood feeding}

Twenty-four hours prior to blood feeding, mosquitoes were starved for 4 hours by removal of sucrose solution. Defibrinated sheep blood (Colorado Veterinarian Product) was mixed with $1 \mathrm{mM}$ ATP and placed in an artificial membrane feeder warmed by a $37^{\circ} \mathrm{C}$ water jacket. Mosquitoes were allowed to feed for 45-60 minutes. Fully engorged mosquitoes were sorted and reared on $10 \%$ sucrose solution and water.

\section{Generating long double-stranded RNA}

Long double-stranded RNA (dsRNA) was generated from Ae. aegypti mosquito total RNA. Primers were designed to amplify an $\sim 500 \mathrm{bp}$ region of the gene of interest (Table S2). cDNA was generated by reverse transcription (RT) using specific reverse primers and SuperScript III 
Reverse Transcriptase (Invitrogen). Polymerase chain reaction (PCR) was performed using specific primers containing a 5, $\mathrm{T} 7$ promotor sequence adapted to both forward and reverse primers and Taq polymerase (NEB). PCR products were purified using the GeneJET PCR Purification kit (Thermo Scientific) and in vitro transcription was performed using the MEGAscript $\mathrm{T} 7 \mathrm{kit}$ (Invitrogen) and incubation at $37^{\circ} \mathrm{C}$ for 12 hours. Following incubation, the product was heated to $75^{\circ} \mathrm{C}$ for 5 minutes and slowly cooled to room temperature for 4 hours to dsRNA annealing. Next, dsRNA was treated with DNase (NEB) and purified by phenolchloroform extraction followed by ethanol precipitation and the purified dsRNA was stored at $80^{\circ} \mathrm{C}$.

\section{dsRNA knockdown of AaFAS1 in Ae. aegypti moquitoes}

dsRNA was introduced via intrathoracic (IT) injection of adult females at 3 to 4 days posteclosion (31). Mosquitoes were anesthetized at $4{ }^{\circ} \mathrm{C}$ on a cold plate. Glass needles were prepared with a vertical pipette puller (P-30, Sutter Instrument Co., Novato, CA) and mosquitoes were IT injected with $3 \mu \mathrm{g} / \mu \mathrm{l}$ of dsRNA in an injection volume of $69 \mathrm{nl}$, twice (total of $\sim 400 \mathrm{ng}$ of dsRNA) using a Nanojet II (Drummond Scientific Company, Broomall, PA). Injected mosquitoes were fed on sucrose solution or blood and reared at $28^{\circ} \mathrm{C}, 80 \%$ relative humidity for 17 days post-injection.

\section{dsRNA knockdown of AaFAS1 gene and DENV2 infection of Aag2 cells}

dsRNA KD was performed in RNA interference-competent Ae. aegypti (Aag2) cells. Aag2 cells were cultured in Schneider's insect medium (Sigma-Aldrich) supplemented with $2 \mathrm{mM}$ Lglutamine, $1 \%$ non-essential amino acids and 10\% FBS. The cells were seeded in a 48 -well plate 
at 50,000 cells/well for 24 hours, and subsequently transfected with $260 \mathrm{ng}$ of dsRNA mixed with TransIT-2020 Reagent (Mirus) following the manufacturer's protocol. New medium with $2 \%$ FBS was replaced at 6 hours post-transfection. Cell viability assays were performed at 2 days post-transfection using resazurin assay.

KD cells were infected with infectious DENV2 expressing a luciferase reporter (DENLuc) supplied by C. Rice, Rockefeller University. Cell culture medium was replaced with $300 \mu 1$ of DEN-Luc supernatant at 48 hours post dsRNA transfection, and cells were incubated at $28^{\circ} \mathrm{C}$ without $\mathrm{CO}_{2}$. Virus supernatant was removed at 24 hours post-infection, and cells were lysed, and luciferase activity was read using the Luciferase Assay System (Promega) as per manufacturer protocol.

\section{Gene expression analyses}

Total RNA was extracted from dissected midgut or whole mosquito by TRIzol (Life Tech) and cDNA was produced via reverse transcription using random primers (Life Tech) and SuperScript III Reverse Transcriptase (Invitrogen). Approximately $400 \mathrm{ng}$ of total cDNA was employed for quantitative PCR (qPCR) analyses. Gene-specific primers are listed in Table S3. $\beta$-actin was used as a reference gene. Relative $A a \mathrm{FAS}$ gene expression was assessed by normalization to the

levels of the $\beta$-actin gene $\left(2^{-\Delta C t}\right)$. The comparative $\mathrm{Ct}\left(2^{-\Delta \Delta C t}\right)$ method was used to calculate the relative expression of $A a \mathrm{FAS}$ following treatment compared to the control (32).

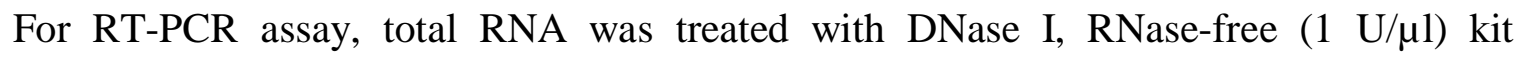
(ThermoFisher) prior to reverse transcription reaction. Purified cDNA was then amplified using Q5® High-Fidelity DNA Polymerase kit (New England BioLabs) with following condition, 
$98^{\circ} \mathrm{C}$ for 30 seconds, 35 cycles of $98^{\circ} \mathrm{C}$ for 10 seconds, $68^{\circ} \mathrm{C}$ and $72^{\circ} \mathrm{C}$ for 2 minutes and 30 seconds. primers are listed in Table S4.

\section{Virus infection of Ae. aegypti by infectious blood meal}

DENV2 serotype 2 strain Jamaica-1409 (33) was cultured in C6/36 cells. Cells were infected with DENV2 at a multiplicity of infection of 0.01 and incubation at room temperature for 1 hour. Virus supernatant was removed, and infected cells were cultured in $5 \mathrm{ml}$ total volume of L15 medium supplemented with $3 \%$ fetal bovine serum (FBS), $50 \mu \mathrm{g} / \mathrm{ml}$ penicillin-streptomycin, and $2 \mathrm{mM}$ L-glutamine. Media was replaced at 7 days post-infection (dpi) and virus supernatant was harvested on 12-14 dpi and immediately used to prepare the infectious blood meal.

\section{Midgut dissection and plaque titration}

Mosquito tissues were collected at multiple days post-exposure to the virus indicated in the figure legends. Isolated midguts or the mosquito carcass (remainder of the body without midgut) were placed separately into $2 \mathrm{ml}$ safe-lock Eppendorf tubes (Eppendorf) containing $250 \mu \mathrm{l}$ of mosquito diluent $(1 \times$ PBS supplemented with $20 \%$ FBS, $50 \mu \mathrm{g} / \mathrm{ml}$ Penicillin/Streptomycin (Gibco), $50 \mu \mathrm{g} / \mathrm{ml}$ Gentamycin (Gibco), and $2.5 \mu \mathrm{g} / \mathrm{ml}$ Amphotericin B (Gibco)) and a stainlesssteel bead (34). Tissue was homogenized using a Retsch Mixer Mill MM400 at 24 cycles per second for $1 \square$ minute, centrifuged at $15,000 \mathrm{~g}$ for $5 \square$ minutes at $4^{\circ} \mathrm{C}$ and supernatant was transferred to a new tube for plaque titration.

Plaque assay was performed on BHK-15 cells. Ten-fold serially diluted viral supernatant was absorbed on the confluent cell layer. After 45 minutes of absorption, cells were overlaid with 1x Minimum Essential Media (MEM), 1X agar supplemented with $2.5 \% \mathrm{FBS}, 25 \mu \mathrm{g} / \mathrm{ml}$ 
Penicillin/Streptomycin, $25 \mu \mathrm{g} / \mathrm{ml}$ Gentamycin, and $1.25 \mu \mathrm{g} / \mathrm{ml}$ Amphotericin B and the cells were incubated at $37^{\circ} \mathrm{C}$ with $5 \% \mathrm{CO}_{2}$. Cells were stained with $0.033 \%$ neutral red (Sigma) in $1 \mathrm{x}$ PBS on day 5 post-infection and plaques were counted at 24 hour post-staining.

\section{Results}

\section{Molecular Characterization of Ae. aegypti FAS genes}

Seven putative AaFAS genes models were obtained via manual annotation using the AaegL5 assembly (35). Previously, five candidate FAS genes (AaFAS1-5), were identified based on the AaegL3 assembly of Nene et al., 2007 (23, 36), and of these, only AaFAS1 and 2 have undergone functional studies (12). The AaegL5 assembly enabled identification of two additional candidate FAS genes (AaFAS6 and AaFAS-like). The corresponding mRNA sequences showing predicted intron/exon structure and initiation and stop codons are shown in Supplemental File 1. The AaFAS1 gene model revealed a gene structure comprising 11 exons, while $A a \mathrm{FAS} 2$ had 5 exons and AaFAS3-5 had 6 exons (Fig. 1). The incomplete $A a \mathrm{FAS}-$ like and AaFAS6 gene models comprised 2 and 3 exons, respectively.

The gene models for AaFAS1-5 appear to be full length, with an average gene product

2 length of 2,360 amino acids (Table 1). AaFAS1-5 possessed features associated with functional

3 FAS, including an initiation methionine, a stop codon and the functional catalytic motifs

4 (DTACSS, EAH and GSVKS) important for ketoacyl synthesis as described by Beedessee et al.

52015 (26). Additionally, AaFAS1-5 contained the YKELRLRGY motif conserved among the

6 FAS genes of vertebrates and invertebrates, present in the polyketide synthase deshydratase

7 domain (Fig. S1). AaFAS3 lacked 6 amino acid residues in the 3' terminus of exon 6 and a total 
8 of 127 non-synonymous substitutions were identified in this model as compared to its AaegL3

9 counterpart.

The Bayesian inference supported AaFAS1-5 as paralogues, and revealed highest percent

11 amino acid similarity between $A a \mathrm{FAS} 1$ and the $H$. sapiens FAS (human FAS) (Fig. 2). Notably,

12 AaFAS1 clustered in a clade comprising the H. sapiens, Mus musculus, Apis mellifera FAS, the

13 D. melanogaster FAS1 and 2, and an uncharacterized Anopheles gambie FAS (Fig. 2). Similarly,

14 AaFAS2 clustered in a clade with another uncharacterized Anopheles gambiae FAS. In contrast,

15 AaFAS3, 4, 5, 6 and -like clustered at the most branched portion of the tree, suggesting a recent

16 diversification event. Phylogenetic analyses and amino acid alignment supported AaFAS1-5 as

17 the counterparts of the AaegL3 genome assembly-derived gene models as follows:

18 LOC5568814-AAEL001194; LOC5570229-AAEL008160; LOC5573929-AAEL022506;

19 LOC5573931-AAEL002237 \& LOC5573927-AAEL002228 (Fig. 2, Table 1). AaFAS-like and

20 AaFAS6 (LOC110675236 \& LOC5573930) were not identified in the AaegL3 assembly

21 suggesting these models are unique to the AaegL5 assembly.

To investigate putative functional domains, AaFAS sequences were aligned to the human

23 FAS using Clustal Omega (27). Human FAS contains seven catalytic domains and three

24 noncatalytic domains (1). Collectively, AaFAS posssesed less than $50 \%$ amino acid identity to

25 human FAS, and of the seven gene models, AaFAS1 had the highest amino acid identity (45.3\%)

26 (Table 1 and Table S5). Alignment of FAS domains also showed modest sequence identity

27 between human FAS and AaFAS (23.03-63.56\%) with greatest similarity for AaFAS1 domains

28 (Table S5). The linear organization of mammalian FAS domains annotated by Maier et. al.,

292008 , is shown in Fig. 3 (1). Conservation in linear organization of motifs associated with known

30 functional domains identified using Pfam 31.0 software is shown in Fig. 3B. Dotted lines 
31 between Fig. 3A and B compare mammalian FAS domains (Fig. 3A) and AaFAS domains (Fig.

32 3B). Pfam analysis did not show the presence of functional methyltransferase domains in $A a$ FAS

33 (Fig. 3B) and protein sequence alignment using Clustal Omega showed deletion within pseudo-

34 methyltransferase ( $\Psi \mathrm{ME})$ domains of AaFAS compared to human FAS (16.20-23.03\% identity;

35 Table S5 and Fig. S2).

36 The gene model of AaFAS-like was 800 amino acids in length and contained all

37 functional catalytic motifs, whereas AaFAS6 was 1,386 amino acids in length, and lacked 38 catalytic motifs but contained the conserved 3' motif YKELRLRGY conserved in FAS (Fig. S1).

39 AaFAS-like contains ketoacyl synthase, ketoacyl synthase_C and ketoacyl-synthase C-terminal 40 extension domains, the first 5' domains of AaFAS1-5 and human FAS (Fig. 3B), while AaFAS6

41 contains $\mathrm{ADH}$ zinc, $\beta$-ketoreductase, $\mathrm{PP}$ binding and thioesterase domains, the last four domains

42 located 3' in AaFAS1-5 and human FAS (Fig. 3B). In the AaegL5 assembly, AaFAS-like and 6

43 are located on chromosome 2 at positions $429280401-429282870$ and $429275401-429279876$,

44 respectively. It is possible that $A a$ FAS-like and -6 reflect an error in genome assembly, or a gene

45 duplication. However, molecular data and the inability to detect transcripts associated with either 46 locus, suggest (Fig. S3) that AaFAS-like and 6 represent pseudogenes (Fig. 3B).

\section{FAS expression during Ae. aegypti development}

49 Mosquitoes undergo four developmental stages: egg, larva, pupa and adult. RT-PCR was used to 50 explore the hypothesis that expression patterns of $A a \mathrm{FAS}$ genes vary among these stages. Five 51 individual mosquitoes were collected for each of the $4^{\text {th }}$ larval instar, pupa and adult stages.

52 Expression of each of the AaFAS genes was normalized to the average expression of the $\beta$-actin 53 gene $\left(2^{-\Delta \mathrm{Ct}}\right)($ Fig. 4). 
Relative expression analyses revealed negligible AaFAS expression in larval and pupal

55 stages, while the highest expression of all genes except AaFAS4 were observed in adult males

56 (Fig. 4). AaFAS1 was the most predominant FAS expressed in adult mosquitoes. Differences in

57 expression levels of any AaFAS were not observed between sugar-fed and 3 days post blood-fed

58 (coinciding with the first gonotrophic cycle) females. The study also revealed negligible AaFAS4

59 expression in all developmental stages and sexes (Fig. 4).

60

\section{Impact of blood feeding on expression of $\mathrm{AaFAS1}$}

62 The diet of the female Ae. aegypti typically involves both nectar and blood. The blood meal is

63 rich in proteins and lipids; therefore, this diet may trigger lipolysis, instead of synthesis, to break

64 down lipid molecules. We compared AaFAS1 expression, the predominant AaFAS in adult

65 females, in sugar-fed females versus blood-fed females (feeding once or twice) (Fig. 5). Blood

66 meals were provided only on specific days as shown in Fig. 5A, while mosquitoes from all

67 groups were fed ad lib on $10 \%$ sugar diet throughout the experiment. Comparisons of AaFAS1

68 gene expression from mosquito samples collected on the same day showed no differences among

69 feeding conditions (Fig. 5B). However, when profiled as ratios (Fig. 5C), we observed a slight,

70 but not significant, reduction of $A a \mathrm{FAS} 1$ expression in females given a single blood-meal as

71 compared to sugar-fed females on days 1, 3 and 4 post-blood meal (pbm) (Fig. 5B: F vs. B, G vs.

$72 \mathrm{C}$ and $\mathrm{H}$ vs. D). This data suggests that diet may only play a minor role, if any, in the expression

73 of AaFAS1 gene. 
76 We hypothesized that the redundancy of AaFAS genes may serve as a backup system for the

77 mosquitoes. To test this hypothesis, we employed AaFAS1 loss-of-function studies to investigate

78 the possibility of compensation by other AaFAS genes. Female mosquitoes were IT injected with

79 dsRNA derived from AaFAS1 or GFP (KD control). On day 2 post-dsRNA injection, five

80 mosquitoes were collected for assessment of AaFAS expression (Fig. 6). We observed an

81 approximate $40 \%$ reduction in $A a \mathrm{FAS} 1$ expression $(\sim 39.3 \pm 13.9 \%)$ in $A a \mathrm{FAS} 1-\mathrm{KD}$ mosquitoes

82 compared to the GFP-KD control (Fig. 6A). In AaFAS1-KD mosquitoes, expression levels of

83 AaFAS2, 3 and 5 and were $191.7 \pm 38.6 \%, 161.4 \pm 21.8 \%$, and $191.1 \pm 38.9 \%$, respectively, in

84 comparison to their levels in GFP-KD control, indicating possible compensation for the loss of

85 AaFAS1 transcript. Conversely, the expression of AaFAS4 was $87.71 \pm 74.0 \%$ compared to

86 AaFAS4 expression in GFP-KD control mosquitoes. To determine whether the upregulation

87 observed in AaFAS2, 3, and 5 could possibly compensate for the loss of AaFAS1 in the

88 AaFAS1-KD mosquitoes, we normalized the level of AaFAS genes to $\beta$-actin. We observed

89 modest expression of $A a \mathrm{FAS}$ transcripts $(5.6 \pm 1.44 \%$ for $A a \mathrm{FAS} 2,4.6 \pm 0.00 \%$ for $A a \mathrm{FAS} 3$, and

$907.07 \pm 0.62 \%$ for $A a$ FAS5 compared to $\beta$-actin), while these upregulation still did not match the

91 remnent of $A a \mathrm{FAS} 1$ expression after the KD effect $(36.1 \pm 11.6 \%)$. This data suggest that other

92 AaFASs may not be able to serve as a backup system for AaFAS1, at least in adult female

93 mosquitoes under transient $\mathrm{KD}$ condition.

94

95 Effect of RNAi-induced AaFAS1 knockdown on DENV2 replication in Ae. aegypti cells

96 Studies in cell culture have shown that FAS activity is required for flavivirus genome replication

$97(13,14,37)$. Biochemical inhibition of FAS activity reduced DENV2 replication in both human 
and mosquito C6/36 cells $(13,15,16)$. The lack of functional RNAi machinery in C6/36 cells hindered the use of transient KD strategy in mosquito cells. However, Ae. aegypti cells, Aag2, have functional RNAi machinery; therefore, we can investigate the role of AaFAS1, the most abundant transcript in female mosquitoes, in DENV2 replication using dsRNA transient KD in these cells (38). At 48 hours post-AaFAS1-KD (time zero of DENV2 infection), the expression level of AaFAS1 in Aag2 cells was $5.15 \pm 6.33 \%$ as compared to AaFAS1 expression in GFPKD control cells (Fig. 7A). At 24 hours post DENV2 infection (72 hours post-KD), we observed significant reduction $(\mathrm{p}<0.001)$ in DENV2 RNA replication in AaFAS1-KD cells as compared to the GFP-KD controls, comparable to replication in DENV2-KD (KD positive control) (Fig. 7B). $\mathrm{KD}$ was not associated with detrimental effects to the cells (Fig. 7C), suggesting that AaFAS1 is required for DENV2 replication in mosquito cells.

\section{Transient inhibition of AaFAS1 reduced DENV2 infection in the midgut of Ae. aegypti}

111 To investigate the role of $A a \mathrm{FAS} 1$ in DENV2 replication in vivo, mosquitoes were IT injected with dsRNA derived from AaFAS1 or GFP genes, and subsequently exposed to DENV2

113 infectious blood meal two-days post-injection (Fig. 8). On days 0, 3 and 7 pbm (corresponding to

1142,5 and 9 days post-dsRNA injection), whole mosquitoes were collected and analyzed for 115 AaFAS1 gene expression (Fig. 8A). On the day of DENV2 infection by blood meal (2 days postdsRNA injection), the level of AaFAS1 expression was downregulated by $53.73 \pm 27.13 \%$

117 relative to GFP-KD group. On day 3 pbm, AaFAS1 expression recovered to $119.82 \pm 49.43 \%$

118 and was comparable to the AaFAS1 expression level in the GFP-KD control. On day 7 pbm, AaFAS1 was upregulated to $191.69 \pm 50.17 \%$, suggesting a possible over-compensation post 120 KD effect (Fig. 8A). 
Investigation of DENV2-fed mosquitoes showed that, at day $3 \mathrm{pbm}$, we observed

122 significant reduction in percent of DENV2 infected midguts compared to the GFP-KD control

123 (Fig. 8B). The odds ratio for AaFAS1 in AaFAS1-KD mosquitoes compared to GFP-KD

124 mosquitoes on day 3 pbm was 0.20 (95\% confidence interval (CI): 0.06 - 0.60) and for DENV2

125 compared to GFP-KD was 0.03 (CI: 0.01 - 0.13) suggesting fewer mosquitoes were infected

126 with DENV2 in the AaFAS1-KD group compared to the GFP-KD group on day 3 pbm.

127 However, no differences in percent infection were observed between AaFAS1-KD and control

128 mosquitoes on days 7 and $14 \mathrm{pbm}$. Infectious particles produced from midguts (virus titer) from

129 AaFAS1-KD and DENV2-KD groups were significantly different from GFP-KD group when the

130 uninfected samples were included in the analysis using the nonparametric Kruskal-Wallis test

131 followed by Dunn's test, with $\mathrm{p}$-values adjusted with the Bonferroni method $(p=0.0002$ and $p<$

1320.0001 , respectively). However, if the titers of uninfected midguts were excluded, differences in

133 virus titer among different dsRNA treatments were not detected (tested by one-way ANOVA

134 followed by Dunn's test; virus titer AaFAS1-KD: $2.40 \times 10^{2}$, GFP-KD: $2.18 \times 10^{3}$, and DENV2-

$135 \mathrm{KD}: 6.43 \times 10^{2}$ plaque forming unit (PFU/midgut).

136 The inhibitory effect of $A a \mathrm{FAS} 1-\mathrm{KD}$ on DENV2 infection did not persist in the midgut

137 beyond day $3 \mathrm{pbm}$. The titer and percent infection in the AaFAS1-KD mosquitoes were

138 comparable to the GFP-KD mosquitoes on day 7 pbm (AaFAS1-KD: 2.70x10 ${ }^{3}$, GFP-KD:

$1392.95 \times 10^{4}$ and DENV2-KD: 5.88 $\times 10^{3}$ PFU/midgut; Fig. 8C). No differences in viral titer and

140 percent infection were observed in midgut on day $7 \mathrm{pbm}$. To investigate whether transient

141 AaFAS1-KD could disrupt virus dissemination, mosquito carcasses (whole body without midgut)

142 were tested on day 14 pbm for virus infection (AaFAS1-KD: $6.57 \times 10^{4}$, GFP-KD: $8.47 \times 10^{4}$ and

143 DENV2-KD: 0.00 PFU/carcass; Fig. 8D). Although we observed no statistical differences in 
144 mean titer in AaFAS1-KD as compared to GFP-KD control samples, two distinct populations of

145 mosquitoes with viral titers in AaFAS1-KD carcasses were observed (Fig. 8D); some with viral

146 titers comparable to GFP-KD control (5.77 $\times 10^{4}$ PFU/carcass; i) and some with distinctively

147 lower titers $\left(2.95 \times 10^{1} \mathrm{PFU} /\right.$ carcass, ii). This observation suggsts that transient KD of $A a \mathrm{FAS} 1$

148 had a prolonged effect that can impact dissemination of DENV2 in mosquitoes.

\section{Discussion}

151 Lipids are essential for a variety of physiological processes in mosquitoes $(3,10,12,39,40)$.

152 Mosquitoes not only acquire lipids from maternal (i.e. deposition to eggs) and dietary sources,

153 but they also have the ability to synthesize lipids de novo. In this study, we characterized the

154 expression of the AaFAS gene family, the key enzyme in the de novo lipid biosynthesis pathway.

155 Additionally, we characterized the response of AaFAS1 expression during infection of 156 mosquitoes and mosquito cell lines with DENV2 to investigate the potential role of this enzyme

157 in supporting virus replication in the mosquito vector.

Seven putative AaFAS genes (AaFAS1-6 and AaFAS-like), were identified in the

AaegL5 assembly based on amino acid similarity to FAS from vertebrates, invertebrates and yeast. Amino acid sequence alignments revealed low amino acid similarity $(<50 \%)$ of $A a$ FAS

161 compared to human FAS (Table 1).

162 The AaFAS1-5 possess seven catalytic and two of the three noncatalytic domains 163 identified in the human FAS annotated using Pfam (Fig. 3) (1, 29). All AaFAS lack the YME 164 domain observed in Drosophila melanogaster (fruit fly), Bombyx mori (silkworm), Apis 165 mellifera (honey bee), Culex pipiens, and Anopheles gambiae (1). Similarly, mammalian FAS 166 also loses the conservation of the motif involved in S-adenosyl-methionine (SAM)-dependent 
167 methyltransferases from the $\Psi \mathrm{ME}$, resulting in an absencce of methyltransferase activity, while

168 it is highly conserved in bacteria and fungi (1). An absence of $\Psi$ ME domain in insect FAS may

169 reflect the unnecessity of this domain.

170 Gene duplication is a hallmark of many mosquito gene families and has been proposed as

171 a source of new evolutionary features $(23,36,41)$. Retention of duplicated genes may be

172 indicative of positive/neutral selection and loci associated with a fitness advantage for the

173 mosquito (42). We performed molecular and prelimnary functional characterization of the

174 AaFAS gene family. We detected transcripts for five of the seven AaFAS genes. We were unable

175 to detect transcripts for either AaFAS6 and AaFAS-like, and conclude that these gene models

176 likely represent pseudogenes or may reflect an issue in the assembly.

177 Since mosquitoes undergo four distinct developmental stages in their life and these stages

178 possess very distinct habitats and food sources, different $A a \mathrm{FAS}$ genes may play roles supporting

179 the unique requirements for FAS in these different life stages. Transcription profiles of $A a$ FAS1-

1805 revealed expression pattern. Notably, we observed low expression levels for all $A a \mathrm{FAS}$ in

181 larval and pupal stages, suggesting that these genes may not be constitutively active across the

182 mosquito life-cycle. We speculate that maternal lipids deposited in eggs during oogenesis (these

183 comprise about $35 \%$ of dry egg weight (7)) and larvae diets may serve to support the metabolic

184 needs during these stages $(8,9,43)$. Thus, they may have minimal requirement for de novo fatty 185 acid biosynthesis.

We observed high levels of expression of all AaFAS, except AaFAS4, in males and to a

187 lesser extent in females. Male Ae. aegypti do not blood feed, but solely obtain their diet from

188 plant nectar, honeydew and fruits (44). Since these diets are high in carbohydrate but low in lipid 
content, high expression of $A a \mathrm{FAS}$ in male mosquitoes may reflect a dependency on $A a \mathrm{FAS}$ for

190 de novo synthesis of lipids.

Expression analyses also revealed AaFAS1 as the dominant AaFAS transcript in both male and female mosquitoes (Fig. 4). The AaFAS1 had the highst amino acid similarity among the AaFAS to the human and mouse FAS genes and is the putative paralogue of these genes.

194 Upon AaFAS1-KD in female mosquitoes, we observed an 2-fold increase in other AaFAS transcripts indicating the attempt to compensate for the loss of $A a \mathrm{FAS} 1$ expression (Fig. 6A). Although we concluded that they fail to compensation for the loss of AaFAS1 since the 197 expression levels of these AaFAS transcripts are still lower than the remaining AaFAS1 expression post-KD, improving the KD efficiency or extending the period of $\mathrm{KD}$ of $A a \mathrm{FAS} 1$

199 (such as using CRISPR-CAS9 knockout) may provide further insights into the redundancy of 200 these $A a \mathrm{FAS}$ genes.

Since previous studies have demonstrated the importance of FAS activity in flavivirus replication in both human and mosquito cells $(13,15,16)$, we wanted to investigate if AaFAS1,

203 also played an important role in DENV2 infection in the mosquito vector. Indeed, KD of 204 AaFAS1 showed significant inhibition of DENV2 infection in midguts of AaFAS1-KD 205 mosquitoes; however, this effect was only observed on day $3 \mathrm{pbm}$. This phenomenon might be 206 caused by the transient KD of $A a \mathrm{FAS}$ expression. Alternative studies with longer suppression of 207 AaFAS1 expression would be required to demonstrate the prominent impact on infection and for 208 further transmission studies.

209 Nonetheless, we found an upregulation of AaFAS1 expression (200\% increase compared 210 to the AaFAS1 levels in the GFP-KD control) on day 9 post-KD. With relevance for strategies 
211 aimed at suppression of host factors for disrupting pathogen transmission,further studies are

212 needed to better understand the biological impact of this "rebound" effect.

213 Additionally, we observed a separation of the virus titers into two groups: high (Fig. 8D,

214 i) and low (Fig. 8D, ii), titers in the carcass of the AaFAS1-KD mosquitoes. Ye et. al. 2015, have

215 shown that when mosquitoes were IT injected with DENV at $10^{6} \mathrm{PFU}$, they expectorated DENV

216 into the saliva at about $10^{2}$ PFU, which is about 100 -fold less than mosquitoes that were IT

217 injected with DENV at $10^{7} \mathrm{PFU}$ (approximate DENV titer in saliva $=10^{4} \mathrm{PFU}$ ) (45). This result

218 suggests that viral titer of DENV in saliva may be dependent on the titer in the body

219 (disseminated titers). In our observations, it is possible that mosquitoes with low body titers

220 (group ii) would inefficienty transmit the virus and the KD of $A a \mathrm{FAS} 1$ may result in a reduction

221 of transmission potential. This study suggests that biological relevance of low viral titres in

222 carcasses and its impact on transmission dynamics is worthy of further investigation.

224 Conclusions

225 Here we pesent expression analyses of the AaFAS gene family and a focused study of the 226 AaFAS1 in Ae. aegypti. We annotated seven AaFAS genes from the AaegL5 genome assembly, 227 and present evidence to support function of five genes. Expression data revealed complexities of $228 A a \mathrm{FAS}$ expression between stages and sexes, and suggest that $A a \mathrm{FAS} 1$ is the dominant transcript 229 in both male and female adult mossquitoes. Sequence homology suggested conservation between 230 mammalian FAS and AaFAS1, and the presence of multiple catalytic domains supports AaFAS1 231 as a key enzyme in de novo lipid biosynthesis. In addition, AaFAS1 was found to facilitate 232 DENV2 replication in both cell culture and in Ae. aegypti. In the latter case, it demonstrated the 233 potential to affect vector competency for virus transmission. 


\section{Supplementary information}

Additional file 1: Figure S1. Alignment of the conserved YKELRLRGY motif. Figure S2.

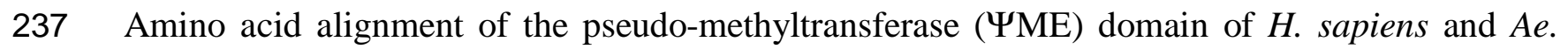
aegypti FAS. Figure S3. RT-PCR assays designed to detect mRNA products of AaFAS-like and AaFAS6. Table S1. List of vertebrate, invertebrate and yeast FAS gene models employed in the present study. Table S2. Primers for generation of dsRNA for knock-down studies. Table S3.

241 Primers for AaFAS expression analyses. Table S4. Primers for RT-PCR assay detecting mRNA

242 products AaFAS-like and AaFAS6. Table S5. Amino acid similarity of FAS domains between $H$.

243 sapiens and Ae. aegypti.

244 Additional file 2: File S1. mRNA and amino acid sequences of 7 AaFAS genes.

246 Abbreviations

247 ACC; acetyl-CoA carboxylase, Ae.aegypti: Aedes aegypti; AaFAS: Aedes aegypti fatty acid 248 synthase; bp: base pair; cDNA: complementary deoxyribonucleic acid; DENV2: dengue virus 249 serotype 2; dpi: days post-infection; dsRNA: long double-stranded ribonucleic acid; FAS: fatty 250 acid synthase; FBS: fetal bovine serum; GFP: green fluorescent protein; IT: intrathoracic; KD:

251 knockdown; MEM: Minimum Essential Media; PBS: phosphate-buffered saline; mRNA: 252 messenger ribonucleic acid; PCR: polymerase chain reaction; quantitative polymerase chain 253 reaction; pbm: post-blood meal; PFU: plaque forming unit; $\Psi \mathrm{ME}$ : pseudo-methyltransferase;

254 RNA, ribonucleic acid; RNAi: interference ribonucleic acid; RT: reverse transcription; RT-PCR:

255 reverse transcription polymerase chain reaction; SNP: single nucleotide polymorphism 


\section{Declarations}

\section{Acknowledgements}

259 The authors thank Kenneth E. Olson and Irma Sanchez-Vargas for providing mosquito eggs. We

260 also thank Rebecca Gullberg, Laura St. Clair, Jeffrey M. Grabowski and Richard J. Kuhn for

261 advice on experimental design, data analysis and critical evaluation of the manuscript and Tach

262 Costello for clerical and administrative support. Graphical Abstract was created using

263 BioRender.

265 Funding

266 This work was funded by R01AI151166 NIH-NIAID and the Boettcher Foundation Early Career

267 Investigator Awards to RP. GR was funded by the R01AI151166 NIH-NIAID. CBS was 268 supported by Purdue internal monies.

269 Supplement.

270

271 Availability of Data and Materials

272 All data and materials were presented in the manuscript and supplementary information.

273

274 Authors' contribution

275 Author Contributions: NC, CBS, GR and EL carried out the experiments. NC, CBS, GR, EL, $276 \mathrm{CAH}$ and RP wrote the manuscript.

\section{Ethics approval and consent to participate}

279 Not applicable 


\section{Consent for publication}

282 All authors consent for publication

\section{Competing interest}

285 The authors have no competing interests.

\section{Author details}

288 Not applicable

\section{References}

291 1. Maier T, Leibundgut M, Ban N. The crystal structure of a mammalian fatty acid synthase.

292 Science. 2008;321(5894):1315-22.

293 2. White SW, Zheng J, Zhang YM, Rock. The structural biology of type II fatty acid 294 biosynthesis. Annu Rev Biochem. 2005;74:791-831.

295 3. Arrese EL, Soulages JL. Insect fat body: energy, metabolism, and regulation. Annu Rev 296 Entomol. 2010;55:207-25.

297 4. Dadd RH, Kleinjan JE. Essential fatty acid for the mosquito Culex pipiens: arachidonic 298 acid. J Insect Physiol. 1979;25(6):495-502.

299 5. Stanley DW, Miller JS. Eicosanoid actions in insect cellular immune functions. 300 Entomologia Experimentalis Et Applicata. 2006;119(1):1-13.

301 6. Ziegler R. Lipid synthesis by overies and fat body of Aedes aegypti (Diptera: Culicidae).

302 European Journal of Entomology. 1997;94:385-91. 
303 7. Ziegler R, Ibrahim MM. Formation of lipid reserves in fat body and eggs of the yellow

304 fever mosquito, Aedes aegypti. Journal of Insect Physiology. 2001;47(6):623-7.

305 8. Atella GC, Shahabuddin M. Differential partitioning of maternal fatty acid and

306 phospholipid in neonate mosquito larvae. J Exp Biol. 2002;205(Pt 23):3623-30.

307 9. Sushchik NN, Yurchenko YA, Gladyshev MI, Belevich OE, Kalachova GS, Kolmakova

308 AA. Comparison of fatty acid contents and composition in major lipid classes of larvae and 309 adults of mosquitoes (Diptera: Culicidae) from a steppe region. Insect Sci. 2013;20(5):585-600.

310 10. Zhou GL, Pennington JE, Wells MA. Utilization of pre-existing energy stores of female

311 Aedes aegypti mosquitoes during the first gonotrophic cycle. Insect Biochemistry and Molecular

312 Biology. 2004;34(9):919-25.

313 11. Jenkin HM, McMeans E, Anderson LE, Yang TK. Comparison of phospholipid 314 composition of Aedes aegypti and Aedes albopictus cells obtained from logarithmic and 315 stationary phases of growth. Lipids. 1975;10(11):686-94.

316 12. Alabaster A, Isoe J, Zhou G, Lee A, Murphy A, Day WA, et al. Deficiencies in acetyl-

317 CoA carboxylase and fatty acid synthase 1 differentially affect eggshell formation and blood 318 meal digestion in Aedes aegypti. Insect Biochem Mol Biol. 2011;41(12):946-55.

319 13. Heaton NS, Perera R, Berger KL, Khadka S, Lacount DJ, Kuhn RJ, et al. Dengue virus 320 nonstructural protein 3 redistributes fatty acid synthase to sites of viral replication and increases 321 cellular fatty acid synthesis. Proc Natl Acad Sci U S A. 2010;107(40):17345-50.

322 14. Martín-Acebes M, Blázquez A, Oya NJd, Escribano-Romero E, Saiz J. West Nile virus 323 replication requires fatty acid synthesis but is independent on phosphatidylinositol-4-phosphate 324 lipids. Plos One. 2011;6(9). 
325 15. Perera R, Riley C, Isaac G, Hopf-Jannasch AS, Moore RJ, Weitz KW, et al. Dengue virus 326 infection perturbs lipid homeostasis in infected mosquito cells. PLoS Pathog. 327 2012;8(3):e1002584.

328 16. Tongluan N, Ramphan S, Wintachai P, Jaresitthikunchai J, Khongwichit S, Wikan N, et 329 al. Involvement of fatty acid synthase in dengue virus infection. Virol J. 2017;14(1):28.

330 17. Welsch S, Miller S, Romero-Brey I, Merz A, Bleck CK, Walther P, et al. Composition 331 and three-dimensional architecture of the dengue virus replication and assembly sites. Cell Host 332 Microbe. 2009;5(4):365-75.

333 18. Junjhon J, Pennington JG, Edwards TJ, Perera R, Lanman J, Kuhn RJ. Ultrastructural 334 characterization and three-dimensional architecture of replication sites in dengue virus-infected 335 mosquito cells. J Virol. 2014;88(9):4687-97.

336 19. Harak C, Lohmann V. Ultrastructure of the replication sites of positive-strand RNA 337 viruses. Virology. 2015;479-480:418-33.

338 20. Chotiwan N, Andre BG, Sanchez-Vargas I, Islam MN, Grabowski JM, Hopf-Jannasch A, 339 et al. Dynamic remodeling of lipids coincides with dengue virus replication in the midgut of 340 Aedes aegypti mosquitoes. PLoS Pathog. 2018;14(2):e1006853.

341 21. Camacho C, Coulouris G, Avagyan V, Ma N, Papadopoulos J, Bealer K, et al. BLAST+: 342 architecture and applications. BMC Bioinformatics. 2009;10:421.

343 22. Giraldo-Calderon GI, Emrich SJ, MacCallum RM, Maslen G, Dialynas E, Topalis P, et

344 al. VectorBase: an updated bioinformatics resource for invertebrate vectors and other organisms 345 related with human diseases. Nucleic Acids Res. 2015;43(Database issue):D707-13.

346 23. Nene V, Wortman JR, Lawson D, Haas B, Kodira C, Tu ZJ, et al. Genome sequence of 347 Aedes aegypti, a major arbovirus vector. Science. 2007;316(5832):1718-23. 
348 24. Larkin MA, Blackshields G, Brown NP, Chenna R, McGettigan PA, McWilliam H, et al.

349 Clustal W and Clustal X version 2.0. Bioinformatics. 2007;23(21):2947-8.

350 25. Corpet F. Multiple sequence alignment with hierarchical clustering. Nucleic Acids Res.

$351 \quad 1988 ; 16(22): 10881-90$.

352 26. Beedessee G, Hisata K, Roy MC, Satoh N, Shoguchi E. Multifunctional polyketide

353 synthase genes identified by genomic survey of the symbiotic dinoflagellate, Symbiodinium

354 minutum. BMC Genomics. 2015;16:941.

355 27. Sievers F, Wilm A, Dineen D, Gibson TJ, Karplus K, Li W, et al. Fast, scalable 356 generation of high-quality protein multiple sequence alignments using Clustal Omega. Mol Syst 357 Biol. 2011;7:539.

358 28. Brown NP, Leroy C, Sander C. MView: a web-compatible database search or multiple 359 alignment viewer. Bioinformatics. 1998;14(4):380-1.

360 29. Mistry J, Bateman A, Finn RD. Predicting active site residue annotations in the Pfam 361 database. BMC Bioinformatics. 2007;8:298.

362 30. Bennett KE, Olson KE, Munoz Mde L, Fernandez-Salas I, Farfan-Ale JA, Higgs S, et al. 363 Variation in vector competence for dengue 2 virus among 24 collections of Aedes aegypti from 364 Mexico and the United States. Am J Trop Med Hyg. 2002;67(1):85-92.

365 31. Mathur G, Sanchez-Vargas I, Alvarez D, Olson KE, Marinotti O, James AA. Transgene366 mediated suppression of dengue viruses in the salivary glands of the yellow fever mosquito, 367 Aedes aegypti. Insect Mol Biol. 2010;19(6):753-63.

368 32. Schmittgen TD, Livak KJ. Analyzing real-time PCR data by the comparative C(T) 369 method. Nat Protoc. 2008;3(6):1101-8. 
370 33. Deubel V, Kinney RM, Trent DW. Nucleotide sequence and deduced amino acid

371 sequence of the structural proteins of dengue type 2 virus, Jamaica genotype. Virology.

$3721986 ; 155(2): 365-77$.

373 34. Merrill AH, Jr., Sullards MC, Allegood JC, Kelly S, Wang E. Sphingolipidomics: high-

374 throughput, structure-specific, and quantitative analysis of sphingolipids by liquid

375 chromatography tandem mass spectrometry. Methods. 2005;36(2):207-24.

376 35. Lee E, Helt GA, Reese JT, Munoz-Torres MC, Childers CP, Buels RM, et al. Web

377 Apollo: a web-based genomic annotation editing platform. Genome Biol. 2013;14(8):R93.

378 36. Matthews BJ, McBride CS, DeGennaro M, Despo O, Vosshall LB. The 379 neurotranscriptome of the Aedes aegypti mosquito. BMC Genomics. 2016;17:32.

380 37. Tang WC, Lin RJ, Liao CL, Lin YL. Rab18 facilitates dengue virus infection by targeting 381 fatty acid synthase to sites of viral replication. J Virol. 2014;88(12):6793-804.

382 38. Sanchez-Vargas I, Scott JC, Poole-Smith BK, Franz AW, Barbosa-Solomieu V, Wilusz J, 383 et al. Dengue virus type 2 infections of Aedes aegypti are modulated by the mosquito's RNA 384 interference pathway. PLoS Pathog. 2009;5(2):e1000299.

385 39. Downer RGH, Matthews JR. Patterns of lipid distribution and utilisation in insect. 386 American Zoologist. 1976;16(4):733-45.

387 40. Zhou GL, Flowers M, Friedrich K, Horton J, Pennington J, Wells MA. Metabolic fate of 388 [C-14]-labeled meal protein amino acids in Aedes aegypti mosquitoes. Journal of Insect 389 Physiology. 2004;50(4):337-49.

390 41. Chau LM, Goodisman MAD. Gene duplication and the evolution of phenotypic diversity 391 in insect societies. Evolution. 2017;71(12):2871-84. 
392 42. Helmkampf M, Cash E, Gadau J. Evolution of the insect desaturase gene family with an 393 emphasis on social Hymenoptera. Mol Biol Evol. 2015;32(2):456-71.

394 43. Dadd RH. Essential Fatty-Acids for the Mosquito Culex-Pipiens. J Nutr. 395 1980;110(6):1152-60.

396 44. Wahid I, Sunahara T, Mogi M. Maxillae and mandibles of male mosquitoes and female 397 autogenous mosquitoes (Diptera: Culicidae). J Med Entomol. 2003;40(2):150-8.

398 45. Ye YH, Carrasco AM, Frentiu FD, Chenoweth SF, Beebe NW, van den Hurk AF, et al. 399 Wolbachia Reduces the Transmission Potential of Dengue-Infected Aedes aegypti. PLoS Negl 400 Trop Dis. 2015;9(6):e0003894. 


\section{Figures}

Fig. 1. Schematic showing the predicted gene structure of the $A a F A S$ gene family. Exons are indicated by yellow cylindrical bars, 5' and 3' non-coding exons by dark grey shading, introns

404 by a black line, start codon by green arrow and stop codon by red T.

Fig. 2. Phylogenetic analysis of AaFAS. Bayesian phylogeny represented with an unrooted tree

407 showing the main relationships between mosquito FAS genes and their counterparts in Ag:

408 Anopheles gambiae, Dm: Drosophila melanogaster, Am: Apis mellifera, Mm: Mus musculus,

409 Hm: Homo sapiens and Sc: Saccharomyces cerevisiae. The branches are supported by posterior

410 probability values. The tree is drawn to scale: amino acid changes per site.

413 Schematic shows linear organization of 7 catalytic and 3 noncatalytic domains of mammalian

414 FAS annotated by Maier et. al., 2008 (1). Seven catalytic domains are shown in big squares and

4153 non-catalytic domains are shown in smaller squares. Abbreviations: KS, $\beta$-ketoacyl synthase;

416 LD, linker; MAT, malonyl-acetyl transferase; DH, dehydratase; ЧME, pseudo-

417 methyltransferase; $\Psi$ KR, pseudo $\beta$-ketoacyl synthase; ER, enoyl reductase; KR, $\beta$-ketoacyl

418 synthase; ACP; acyl carrier protein and TE, thioesterase. (b) Schematics show conserved 419 domains or motifs of FAS genes and their organization annotated using Pfam 31.0 software.

420 Abbreviations are as follows: ketoacyl_synt, $\beta$-ketoacyl synthase; ketoacyl_synt_C, $\beta$-ketoacyl-

421 acyl carrier protein synthase; ketoacyl_assoc, ketoacyl-synthase C-terminal extension;

422 acyl_transf_1, acyl transferase domain; PS-DH, polyketide synthase; methyltransf, 
423 methyltransferase domain; ADH-zinc, zinc binding dehydrogenase; KR, $\beta$-ketoreductase

424 domain; PP-binding, phosphopantetheine attachment site; thioesterase, thioesterase domain.

Fig. 4. Expression of $\mathrm{AaFAS}$ in mosquito developmental stages and sexes. RNA was prepared

427 from 5 specimens of $4^{\text {th }}$ instar larvae, pupae, sugar-fed males, sugar-fed females, and females 3428 days pbm (all adult mosquitoes were collected at the same day time; 8-10 days post eclosion). 429 Samples were subjected to RT-qPCR to assess relative expression of AaFAS1-5 and AaFAS-like. 430 RNA levels between samples were normalized to the $\beta$-actin gene using $2^{-\Delta \mathrm{Ct}}$ method. The boxes 431 show the $25^{\text {th }}$ and $75^{\text {th }}$ percentiles, the whiskers show the minimum and maximum values and the midline indicates the median of the relative gene expression value.

Fig. 5. Expression of AaFAS1 in sugar-fed and blood-fed mosquitoes. (a) Schematic of the

435 experimental design. Mosquitoes were reared in 3 different conditions: sugar-fed only, one blood

436 meal, which were fed on day zero, and two blood meals, which were again fed on day 3.

437 Mosquitoes in all conditions were also allowed access to additional sugar and water at all time

438 throughout the experiment. Five mosquitoes from each condition were collected on different 439 days post $1^{\text {st }}$ blood meal feeding designated in letters A-K. (b) AaFAS1 expression profile (2 $2^{-\Delta \mathrm{Ct}}$; 440 normalized to actin) of each sampling group were shown. Statistical analysis was performed by 441 one-way ANOVA followed by Tukey's multiple comparison tests. *, p<0.05 and **, $\mathrm{p}<0.01$.

442 (c) Expression of $A a \mathrm{FAS} 1$ was measured by the comparative expression $\left(2^{-\Delta \Delta \mathrm{Ct}}\right)$ method. The 443 samples used for comparisons are shown on the $\mathrm{X}$ - axis. Boxes show the $25^{\text {th }}$ and $75^{\text {th }}$ 444 percentiles, whiskers show the minimum and maximum values, and the midline shows the 445 median of the relative gene expression value. 
Fig. 6. Comparive analyses of AaFAS expression in AaFAS1-KD mosquito. Three-day-old adult female mosquitoes were intrathoracically injected with dsRNA derived from AaFAS1 or GFP mRNA sequence (an irrelevant dsRNA control). Mosquitoes were reared on 10\% sugar diet

449 for 2 days post-injection. Three pools of 5 mosquitoes were collected for $A a \mathrm{FAS}$ gene expression 450 measurements. (a) Percent relative expression of AaFAS genes in AaFAS1-KD mosquitoes was 451 compared to GFP-KD control mosquitoes using the comparative $\mathrm{Ct}\left(2^{-\Delta \Delta \mathrm{Ct}}\right)$ method. (b) Gene 452 expression profiles of $A a \mathrm{FAS}$ were measured as normalized to $\beta$-actin gene expression $\left(2^{-\Delta \mathrm{Ct}}\right)$.

453 Boxes show the $25^{\text {th }}$ and $75^{\text {th }}$ percentiles, whiskers show the minimum and maximum values and 454 midline shows median of the relative gene expression value. Statistical analysis was performed 455 by one-way ANOVA followed by Tukey's multiple comparison tests. **, p<0.01, ***, p<0.005 456 and $* * * *, \mathrm{p}<0.001$

461 RNA replication of luciferase-expressing DENV2 in Aag2 cells treated with dsRNA derived 462 from AaFAS1, DENV2 or GFP. Cells were transfected with dsRNA for two days prior to 463 infection with luciferase-tagged DENV2. At $24 \mathrm{hpi}$, cells were lysed and were assayed for 464 luciferase activity (RLU). One-way ANOVA followed by Tukey's multiple comparison test were 465 applied for statistical analysis. ****, p < 0.001. (c) Viability of Aag2 cells treated with dsRNA 466 derived from AaFAS1, DENV2 (positive DENV2 KD control) and GFP (negative DENV2 KD 467 control) genes assessed by resazurin assay. 
469 Fig. 8. Transient KD of AaFAS1 expression by dsRNA temporarily reduced DENV2

470 infection in midguts. (a) Percent AaFAS1 expression in FAS-KD mosquitoes. Mosquitoes were

471 IT injected with $\sim 400 \mathrm{ng}$ of dsRNA derived from AaFAS1, DENV2 or GFP two days prior to

472 blood meal infection. On days 0,3 and 7 pbm (days 2, 5 and 9 days post IT injection), 3 pools of

4735 mosquitoes were collected and analyzed for AaFAS1 expression compared to AaFAS1

474 expression in GFP-KD mosquitoes. (b-d) Mosquitoes were IT injected with dsRNAs and

475 infected with DENV2 by infectious blood meal at 2 days post injection. Plaque assay was

476 performed on midguts dissected on (b) day 3 and (c) day 7 and (d) carcasses (whole body

477 without midgut) collected on day $14 \mathrm{pbm}$. (i) and (ii) indicate the separation of DENV2 titers in

478 the carcass that were produced from the AaFAS1-KD mosquitoes. Mean virus titer (infectious

479 particles) was calculated for infected samples only. One-way ANOVA followed by Dunn's tests

480 were applied to test the differences in virus titer among samples, ${ }^{*}, p<0.05$. The odds ratio was

481 applied to test the differences in percent infection. The significant reduction of percent infection

482 in AaFAS1-KD or DENV2-KD compared to GFP-KD are highlighted in bold. 
484 Table 1. Summary of AaFAS gene family predicted from the Aedes aegypti AaegL5 assembly. The AaegL5 annotation is shown

485 in comparison to the AaegL3 gene models reported by Nene et al., 2007 [23].

\begin{tabular}{|c|c|c|c|c|c|c|c|c|c|c|c|}
\hline Name & $\begin{array}{c}\text { NCBI } \\
\text { Accession } \\
\text { Number } \\
\text { (AaegL5) }\end{array}$ & $\begin{array}{c}\text { VectorBas } \\
\text { e } \\
\text { Accession } \\
\text { number } \\
\text { (AaegL3) }\end{array}$ & $\begin{array}{c}\text { Max } \\
\text { number } \\
\text { of } \\
\text { exons }\end{array}$ & $\begin{array}{c}\text { Chro- } \\
\text { mo- } \\
\text { some }\end{array}$ & Location & $\begin{array}{c}\% \\
\text { Ident } \\
\text { with } \\
\text { human } \\
\text { FAS }\end{array}$ & $\begin{array}{c}\text { Length } \\
\text { (nucleot } \\
\text { ides) }\end{array}$ & $\begin{array}{c}\text { Length } \\
\text { (amino } \\
\text { acids) }\end{array}$ & $\begin{array}{c}\text { No. Splice } \\
\text { variants/ } \\
\text { isoforms }\end{array}$ & $\begin{array}{l}\text { Notes on } \\
\text { the revised } \\
\text { annotation }\end{array}$ & $\begin{array}{c}\% \\
\text { identity } \\
\text { (AaegL3 } \\
\text { \& L5) }\end{array}$ \\
\hline $\begin{array}{l}\text { AaFAS } \\
1\end{array}$ & $\begin{array}{l}\text { LOC55688 } \\
14\end{array}$ & $\begin{array}{l}\text { AAEL0011 } \\
94\end{array}$ & 11 & 2 & $\begin{array}{l}\text { NC_035108.1 } \\
(307544012 . .30760 \\
1765, \text { complement })\end{array}$ & 45.3 & 9732 & 2422 & 1 & $\begin{array}{l}1 \text { SNP in } \\
\text { exon_4 }\end{array}$ & 99.9 \\
\hline $\begin{array}{l}\text { AaFAS } \\
2\end{array}$ & $\begin{array}{l}\text { LOC55702 } \\
29\end{array}$ & $\begin{array}{l}\text { AAEL0081 } \\
60\end{array}$ & 5 & 3 & $\begin{array}{l}\text { NC_035109.1 } \\
\text { (9993811..1000242 } \\
7, \text { complement) }\end{array}$ & 36.7 & 8368 & 2385 & 1 & $\begin{array}{l}1 \text { SNP in } \\
\text { exon_5 }\end{array}$ & 100 \\
\hline $\begin{array}{l}\text { AaFAS } \\
3\end{array}$ & $\begin{array}{l}\text { LOC55739 } \\
29\end{array}$ & $\begin{array}{l}\text { AAEL0225 } \\
06\end{array}$ & 6 & 2 & $\begin{array}{l}\text { NC_035108.1 } \\
(429256663 . .42926 \\
4110, \text { complement })\end{array}$ & 32.9 & 7144 & 2334 & 1 & $\begin{array}{l}18 \text { SNPs } \\
\text { exon_1, } 31 \\
\text { SNPs } \\
\text { exon_2, } 11 \\
\text { SNPs } \\
\text { exon_3, } 52 \\
\text { SNPs } \\
\text { exon_4, 3 } \\
\text { SNPs } \\
\text { exon_5, and } \\
12 \text { SNPs } \\
\text { exon_6. } \\
\text { Deletion of } \\
\text { six amino } \\
\text { acid } \\
\text { residues } \\
\text { when }\end{array}$ & 94.6 \\
\hline
\end{tabular}




\begin{tabular}{|c|c|c|c|c|c|c|c|c|c|c|c|}
\hline & & & & & & & & & & $\begin{array}{l}\text { compared } \\
\text { with the } \\
\text { AaegL3 } \\
\text { orthologue }\end{array}$ & \\
\hline $\begin{array}{l}\text { AaFAS } \\
4\end{array}$ & $\begin{array}{l}\text { LOC55739 } \\
31\end{array}$ & $\begin{array}{l}\text { AAEL0022 } \\
37\end{array}$ & 6 & 2 & $\begin{array}{l}\text { NC_035108.1 } \\
\text { (429327062..42933 } \\
4421, \text { complement) }\end{array}$ & 34.6 & 7068 & 2333 & 1 & $\begin{array}{l}4 \text { SNPs } \\
\text { exon_2, } 10 \\
\text { SNPs } \\
\text { exon_4, } 6 \\
\text { SNPs } \\
\text { exon_6 }\end{array}$ & 99.1 \\
\hline $\begin{array}{l}\text { AaFAS } \\
5\end{array}$ & $\begin{array}{l}\text { LOC55739 } \\
27\end{array}$ & $\begin{array}{l}\text { AAEL0022 } \\
28\end{array}$ & 6 & 2 & $\begin{array}{l}\text { NC_035108.1 } \\
(429228612 . .42923 \\
6010, \text { complement })\end{array}$ & 32.9 & 7097 & 2324 & 1 & $\begin{array}{l}5 \text { SNPs } \\
\text { exon_2, } 2 \\
\text { SNPs } \\
\text { exon_3, } 3 \\
\text { SNPs } \\
\text { exon_4, 1 } \\
\text { SNP exon_6 }\end{array}$ & 99.5 \\
\hline $\begin{array}{l}\text { AaFAS } \\
\text {-like }\end{array}$ & $\begin{array}{l}\text { LOC11067 } \\
5236\end{array}$ & - & 2 & 2 & $\begin{array}{l}\text { NC_035108.1 } \\
\text { (429280401..42928 } \\
2870, \text { complement) }\end{array}$ & 36.1 & 2409 & 800 & 1 & - & - \\
\hline $\begin{array}{l}\text { AaFAS } \\
6\end{array}$ & $\begin{array}{l}\text { LOC55739 } \\
30\end{array}$ & - & 3 & 2 & $\begin{array}{l}\text { NC_035108.1 } \\
\text { (429275401..42927 } \\
9876, \text { complement })\end{array}$ & 36.8 & 4353 & 1386 & 1 & - & - \\
\hline
\end{tabular}


bioRxiv preprint doi: https://doi.org/10.1101/2022.02.14.480456; this version posted February 15,2022 . The copyright holder for this preprint (which was not certified by peer review) is the author/funder. All rights reserved. No reuse allowed without permission. 
bioRxiv preprint doi: https://doi.org/10.1101/2022.02.14.480456; this version posted February 15, 2022. The copyright holder for this preprint (which was not certified by peer review) is the author/funder. All rights reserved. No reuse allowed without permission.

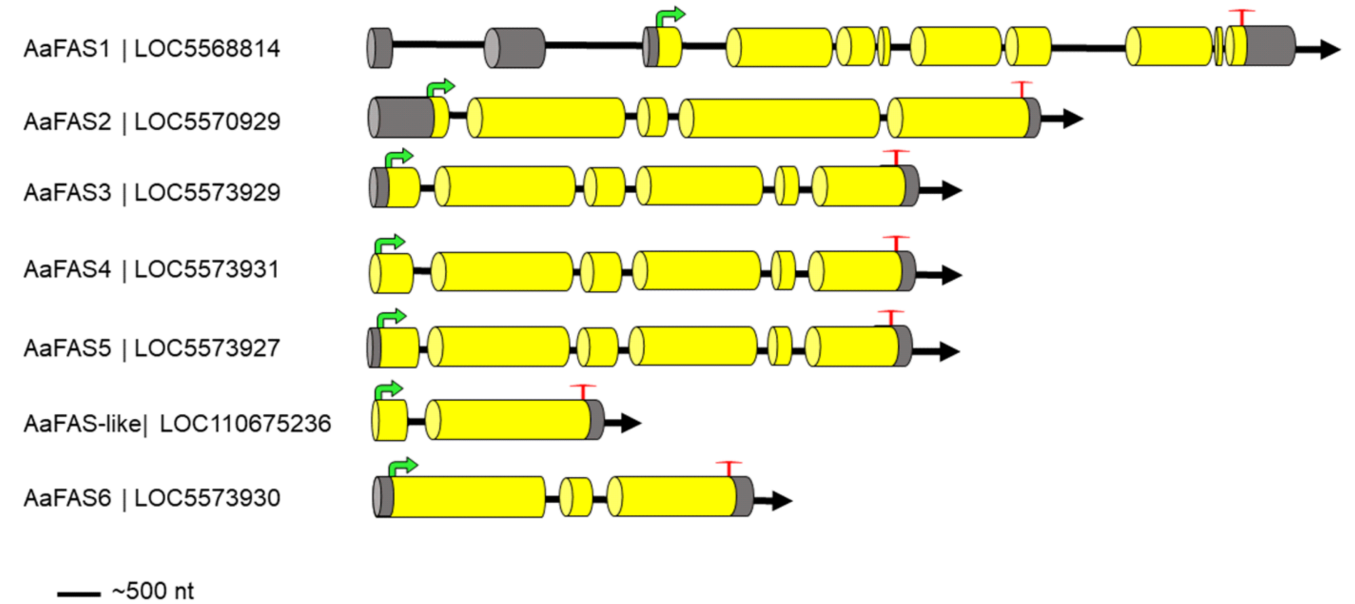


bioRxiv preprint doi: https://doi.org/10.1101/2022.02.14.480456; this version posted February 15,2022 . The copyright holder for this preprint (winch was not certified by peer review) is the author/funder. All rights reserved. No reuse allowed without permission.

$\frac{\pi}{0}$

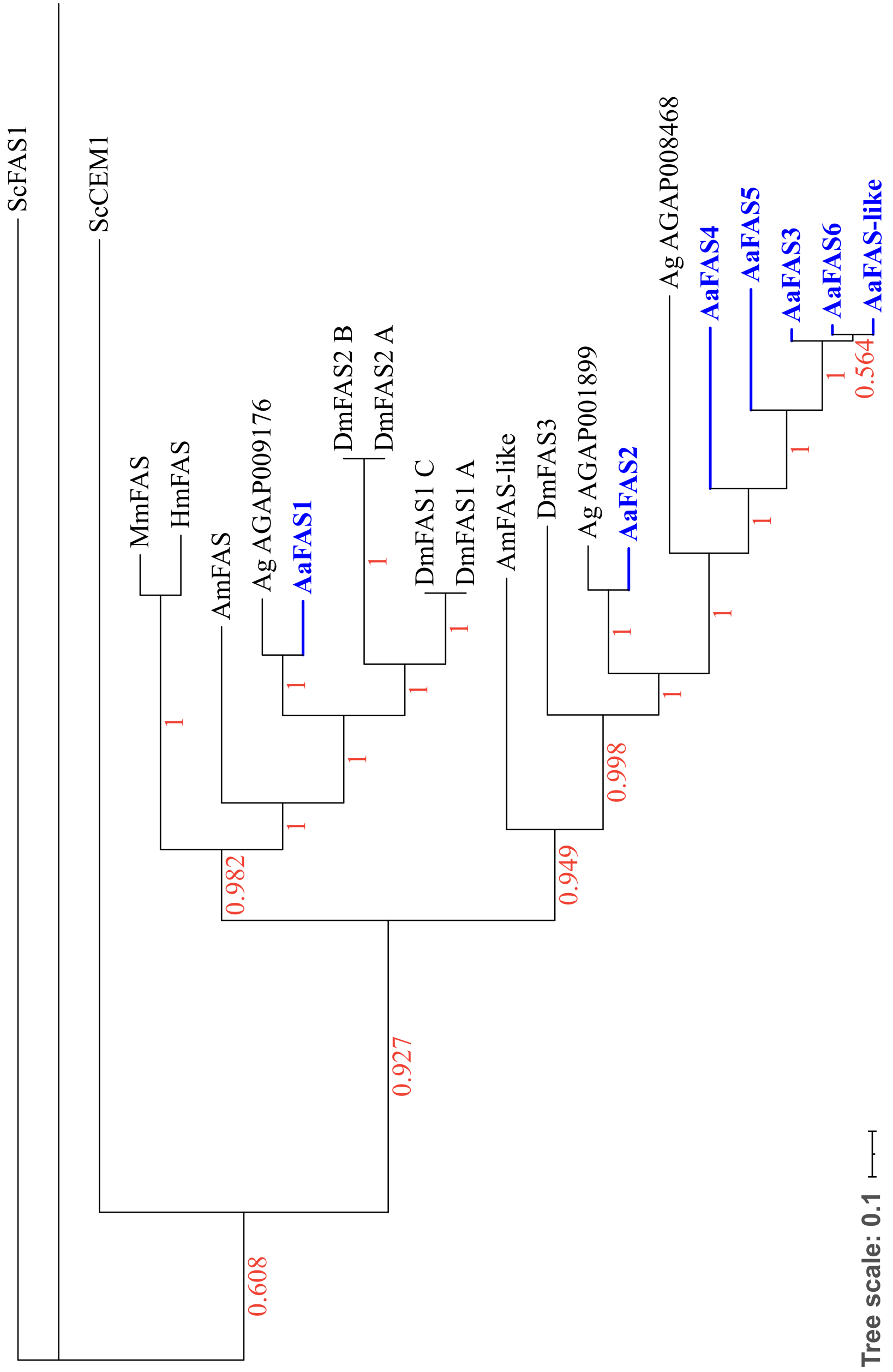


a

b

Human FAS

AaFAS1

AaFAS2

AaFAS3

AaFAS4

AaFAS5

AaFAS-like

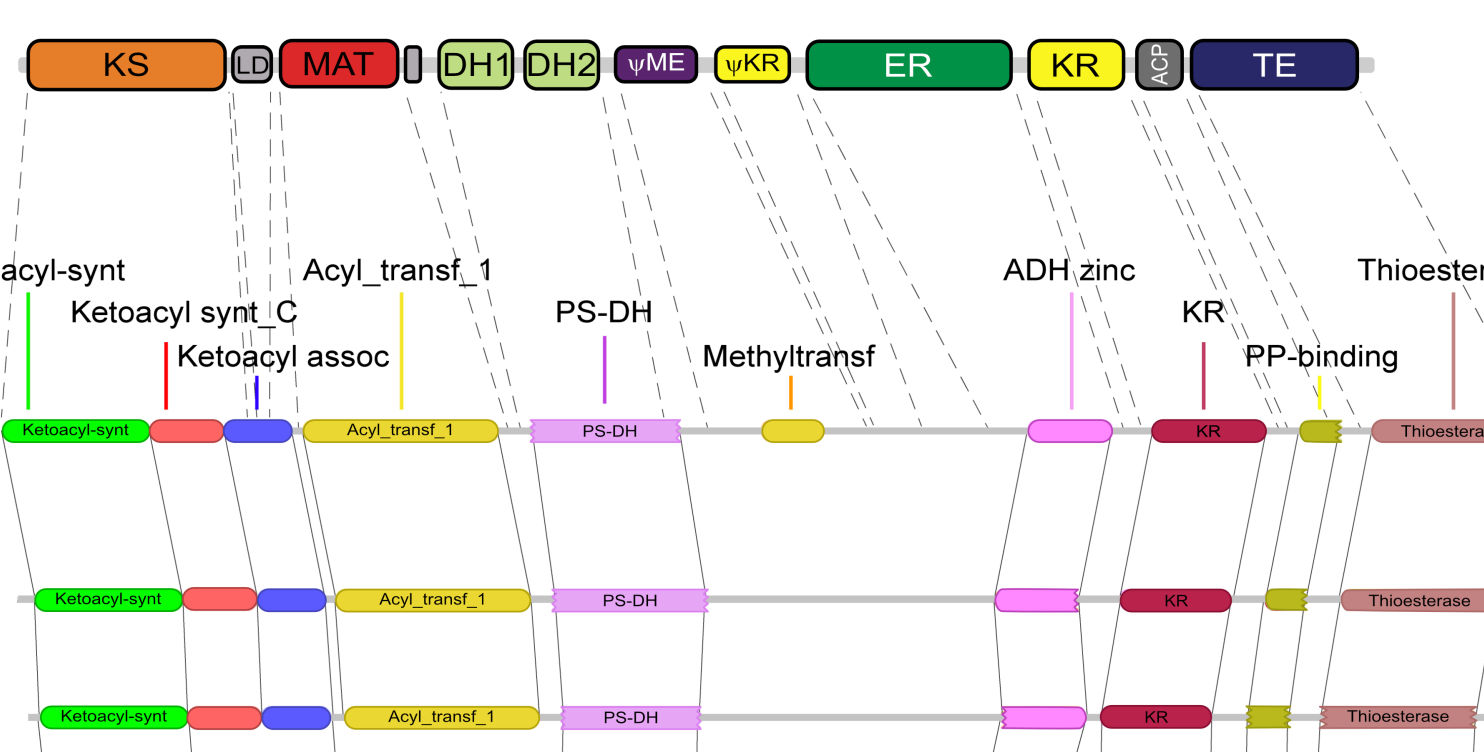

Ketoacyl-synt

I) Acyl_transf_1

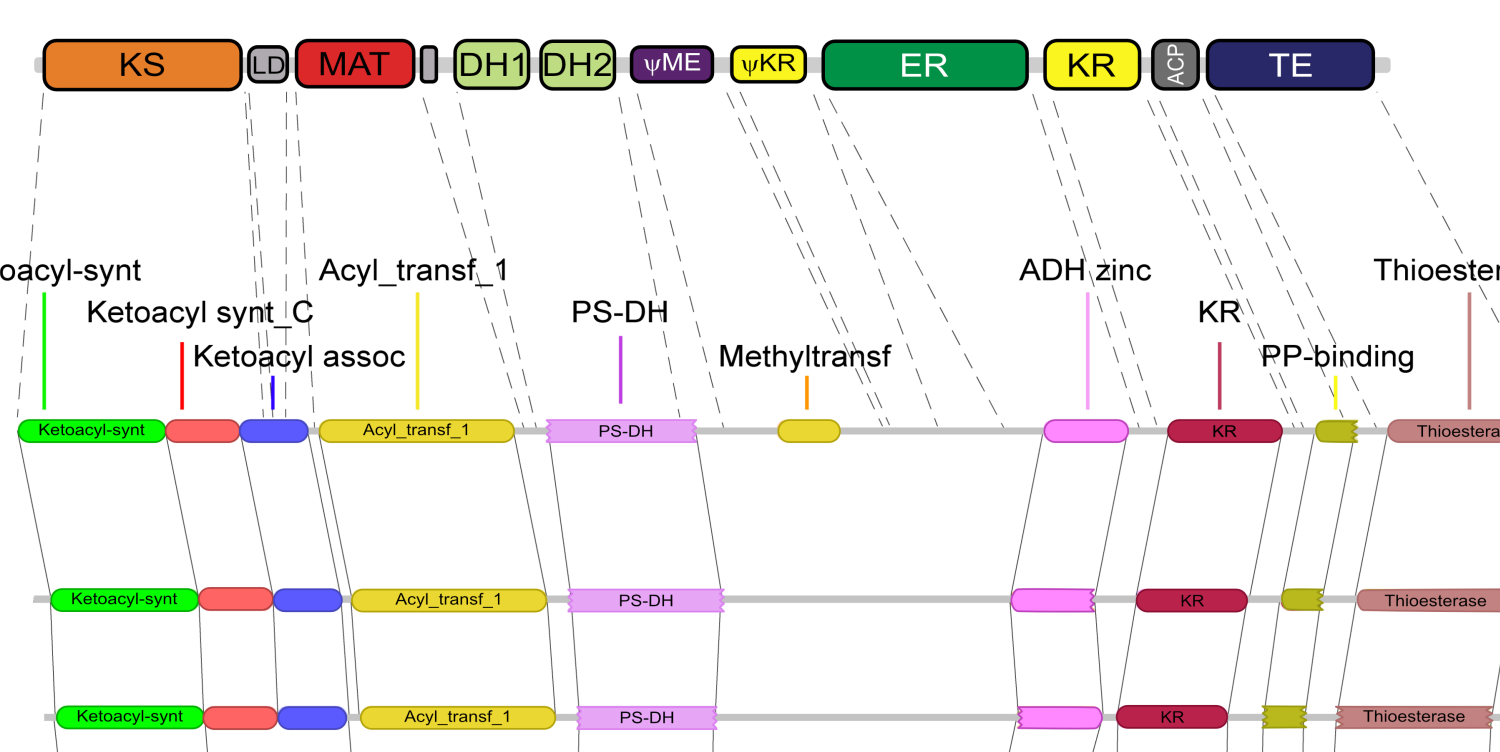

$\mathrm{DH} 2$

YME $\Psi \mathrm{KR}$

ER

KR 0
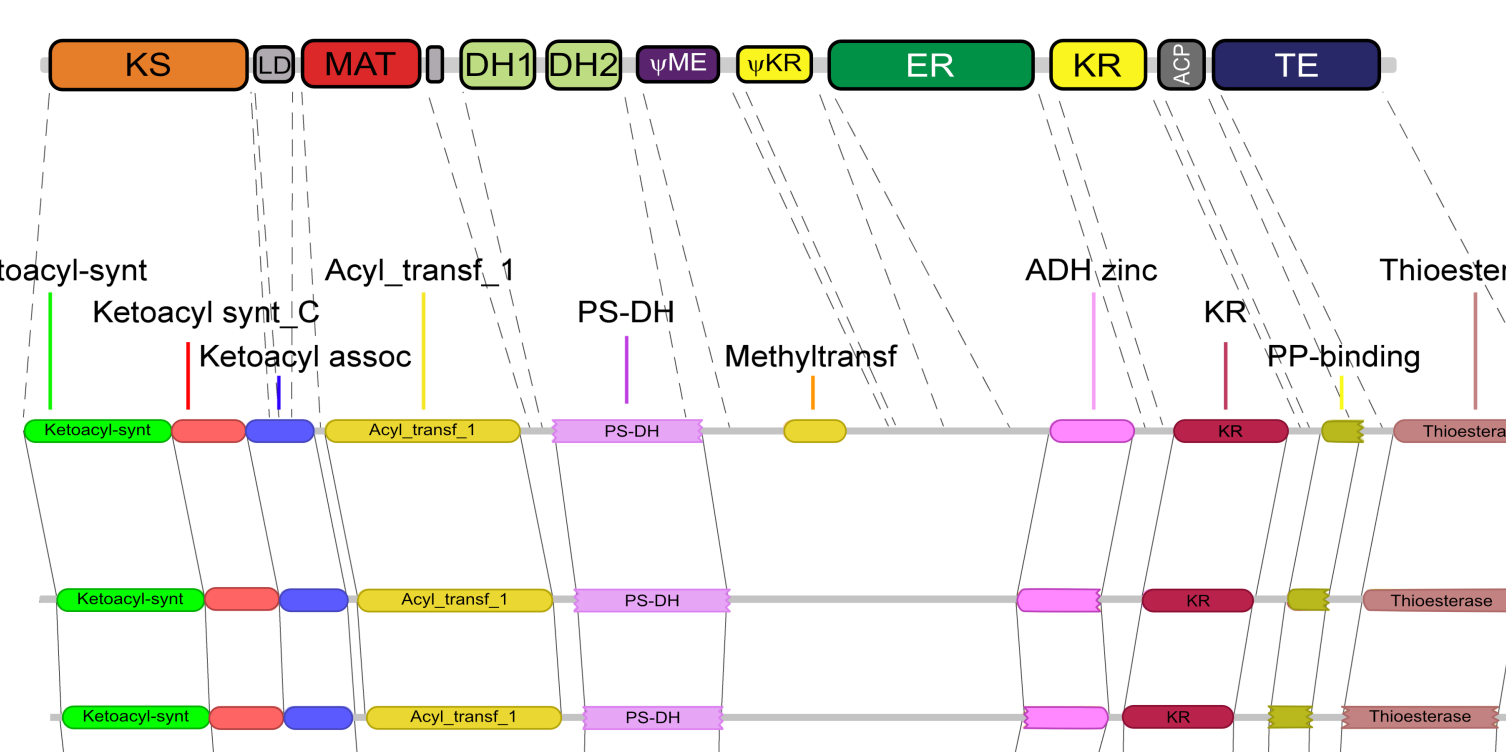

Methyltransf

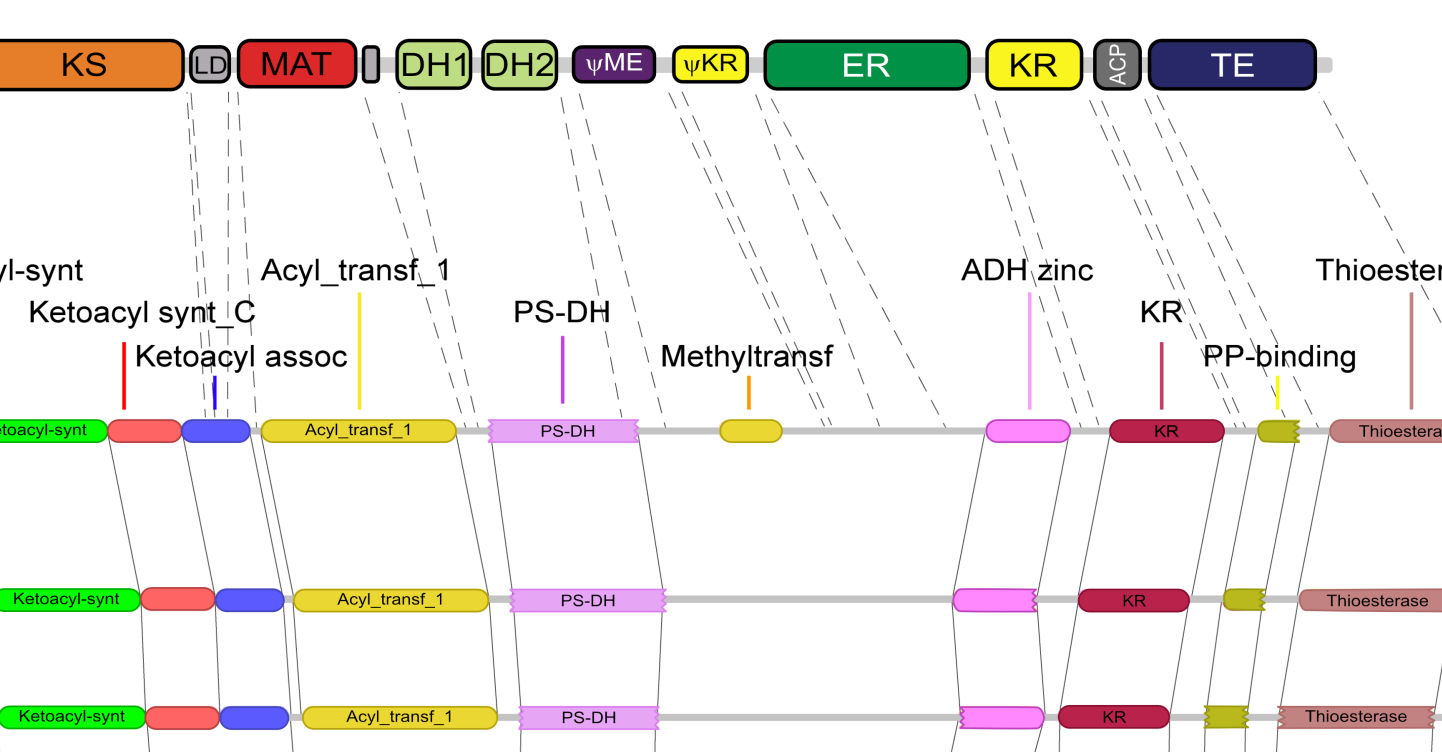

Acyl_transf_1
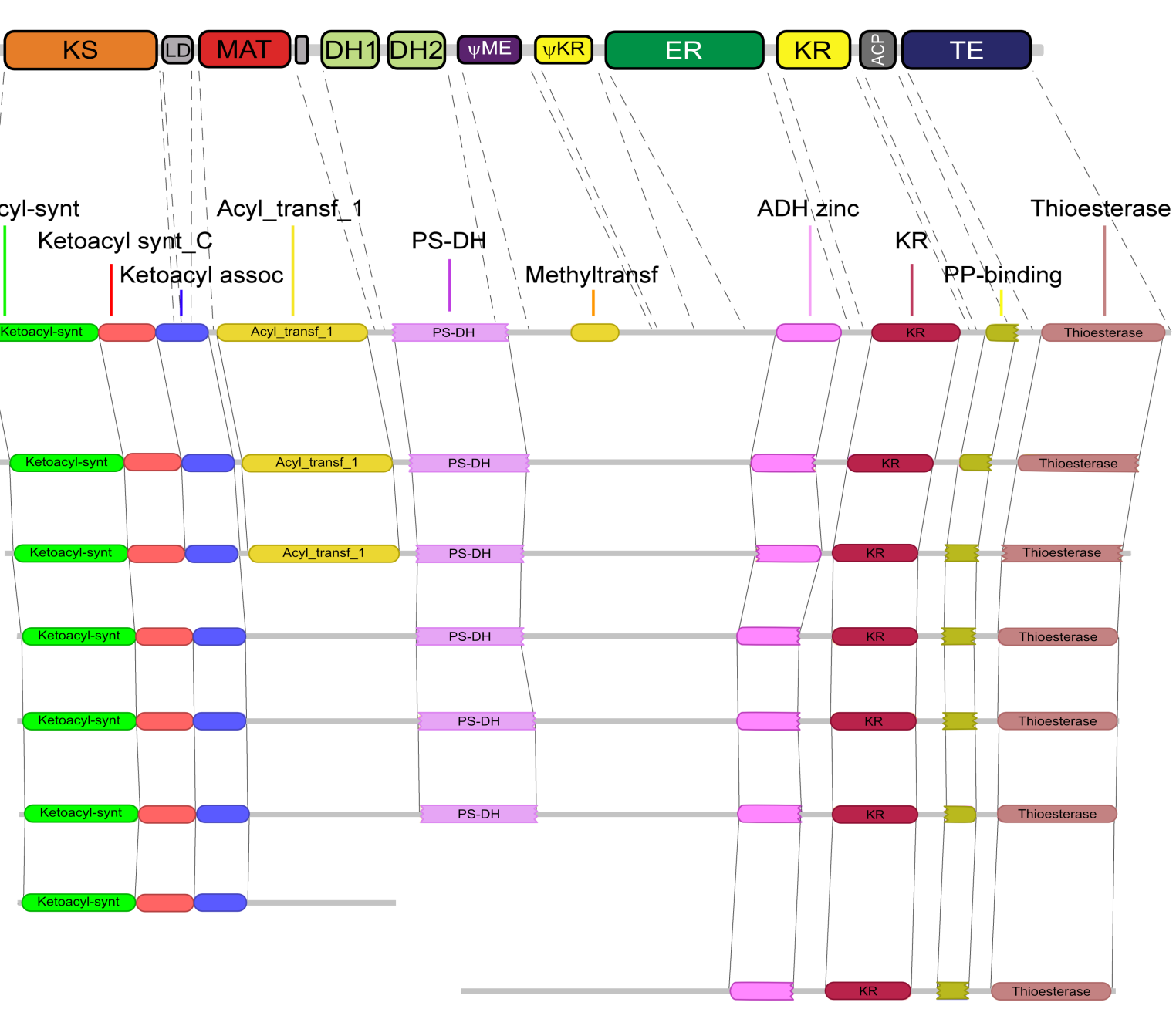

\section{AaFAS6}



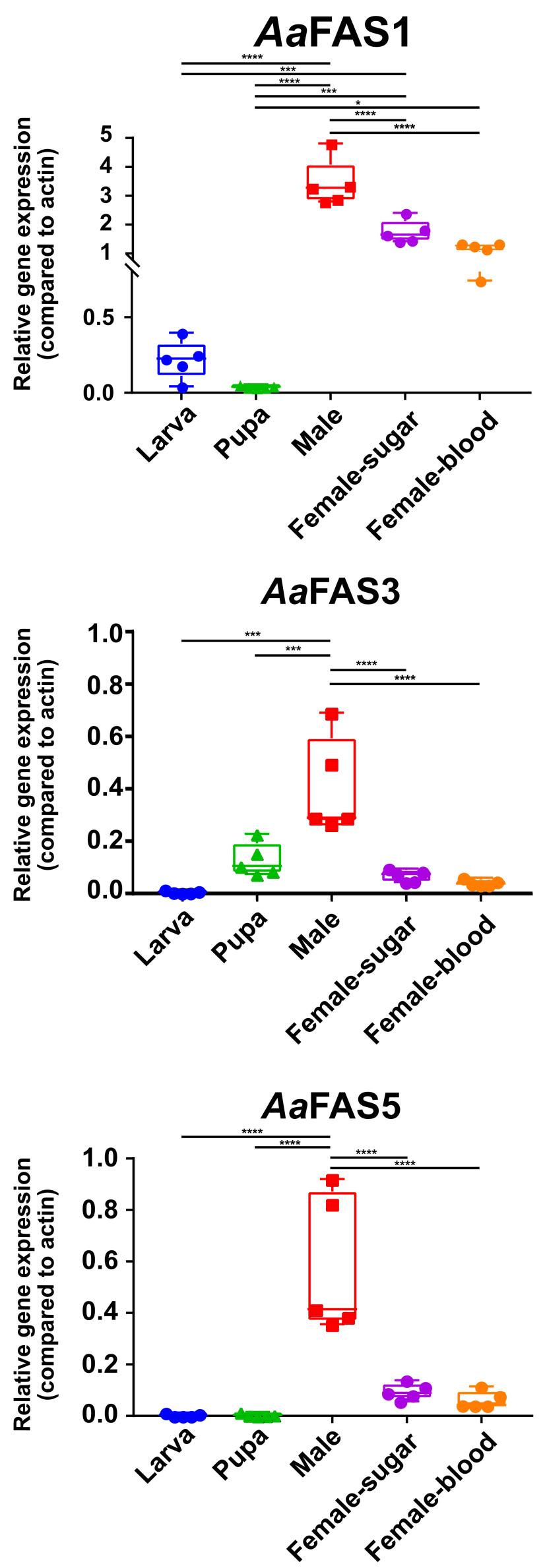

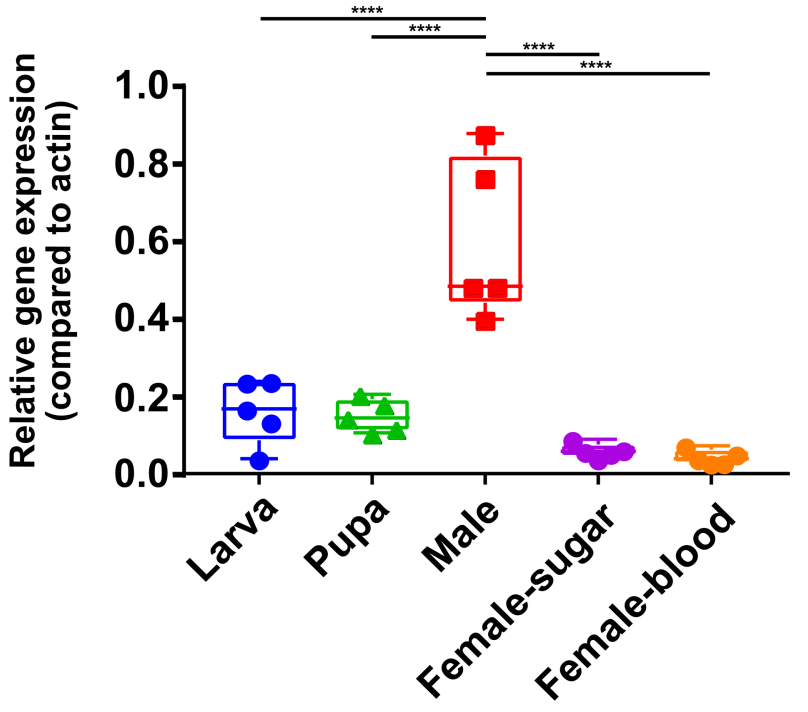

AaFAS4

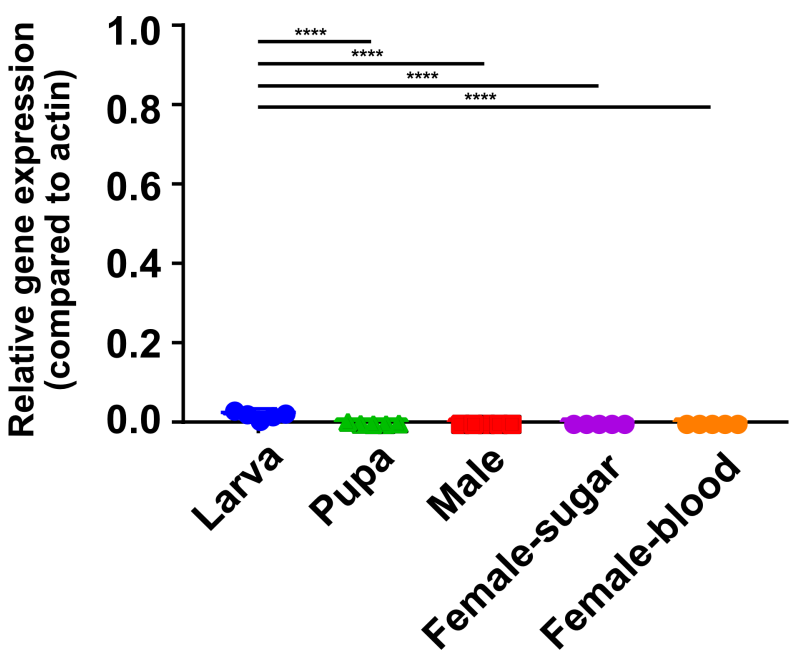


a

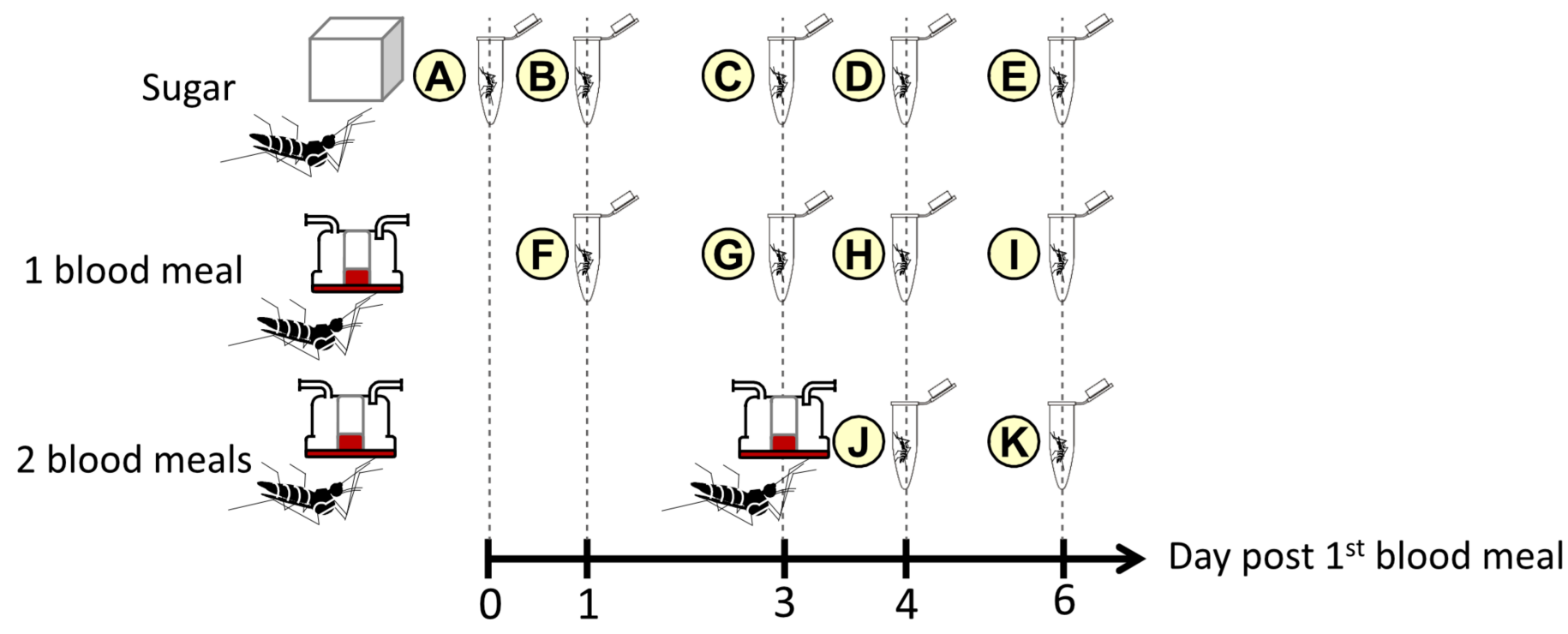

b

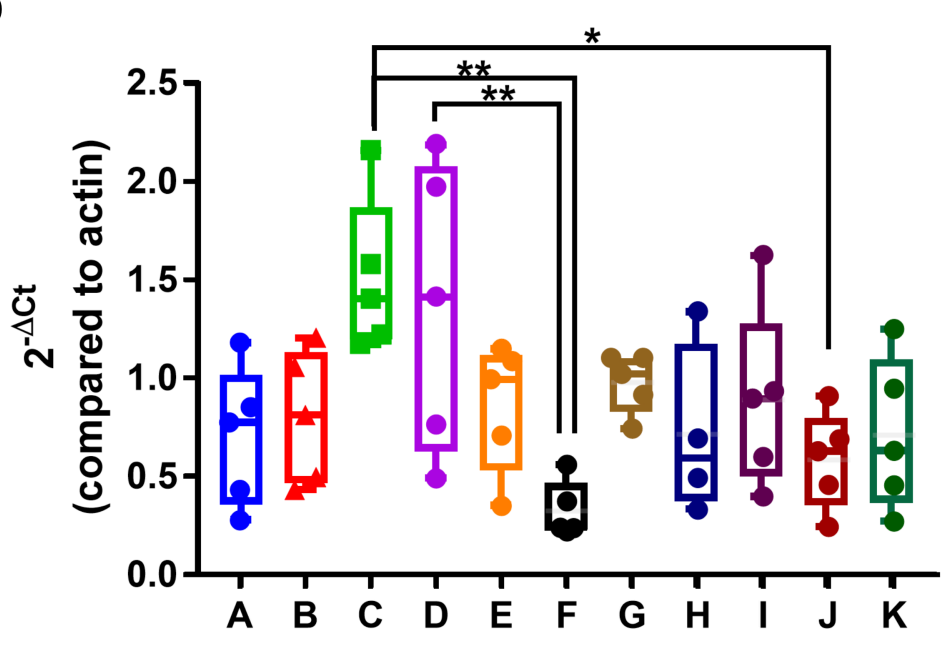

C

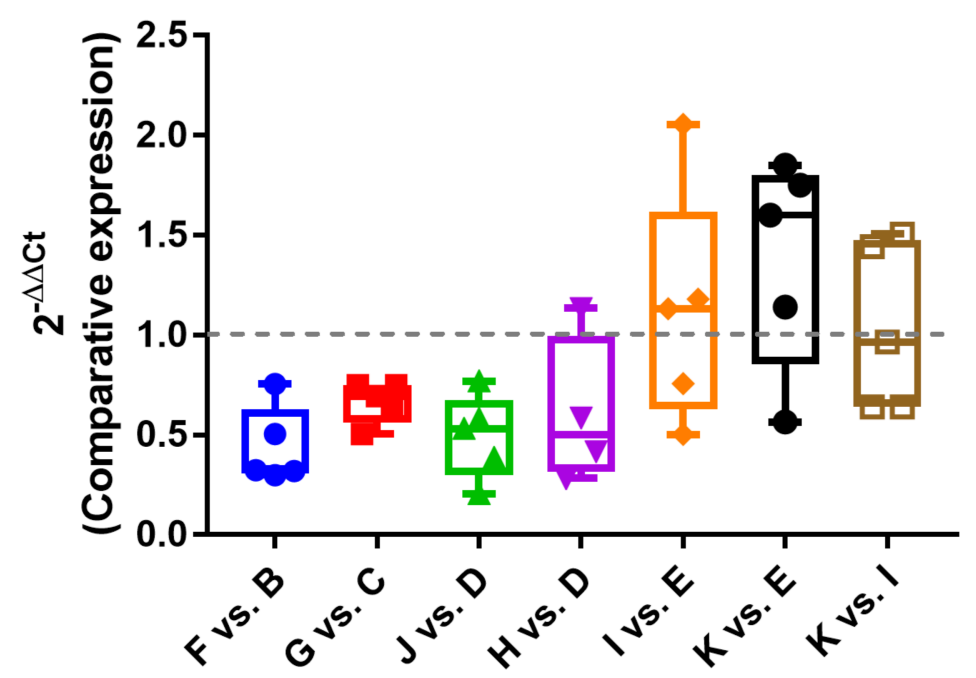


a

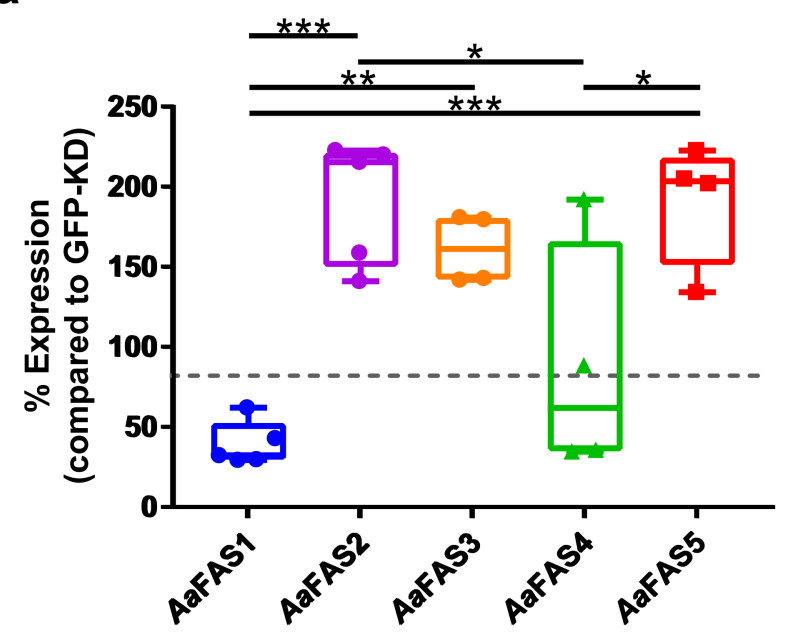

b

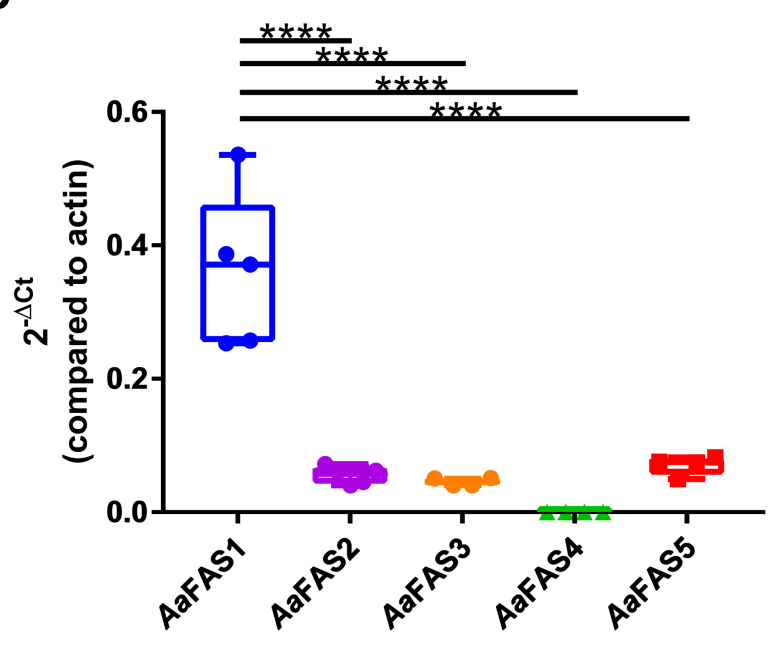


a

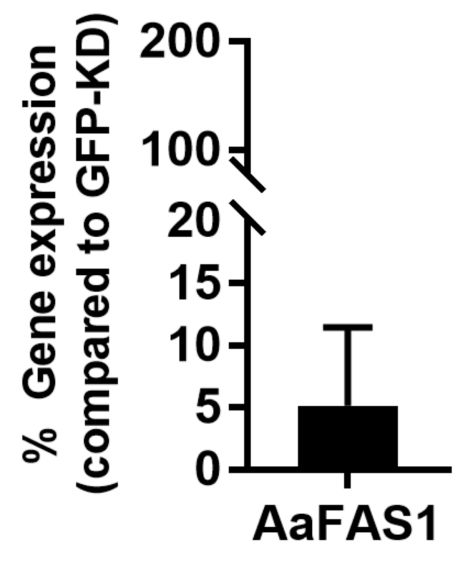

b

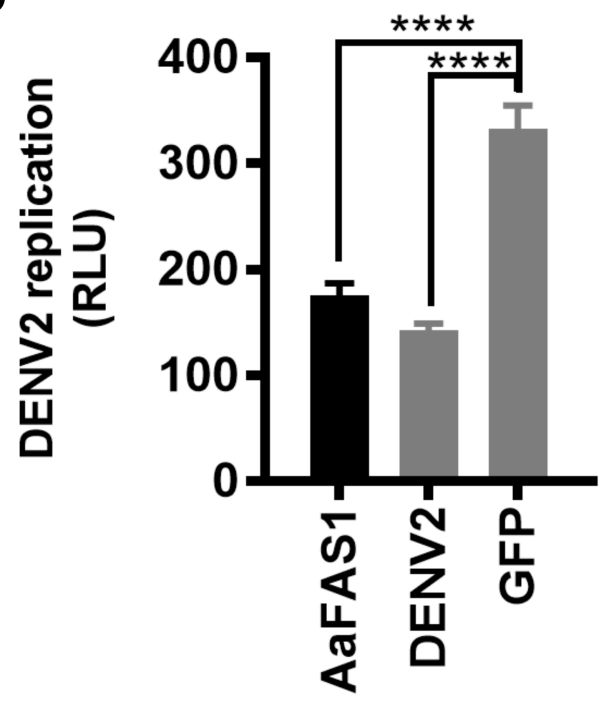

C

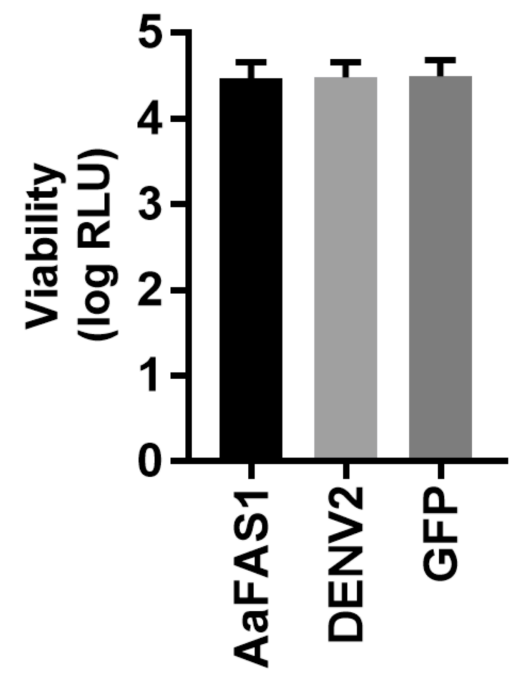


a

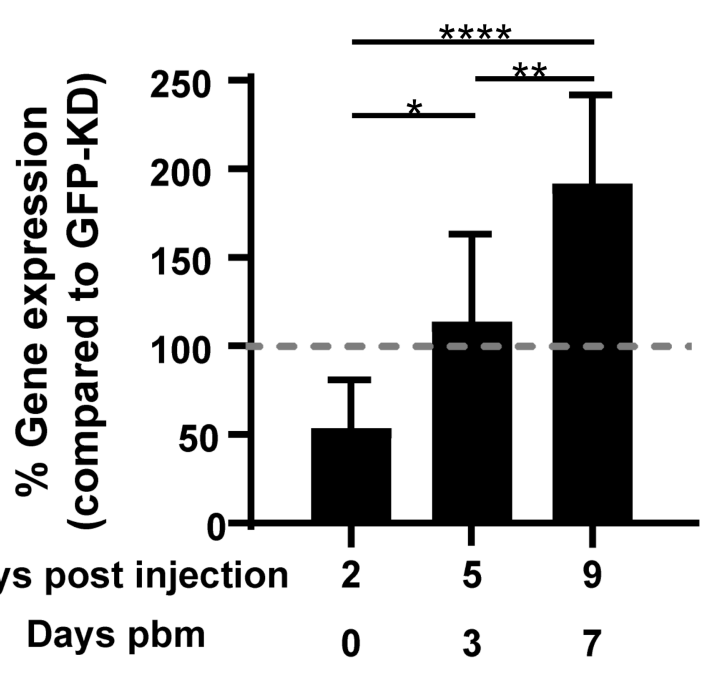

C

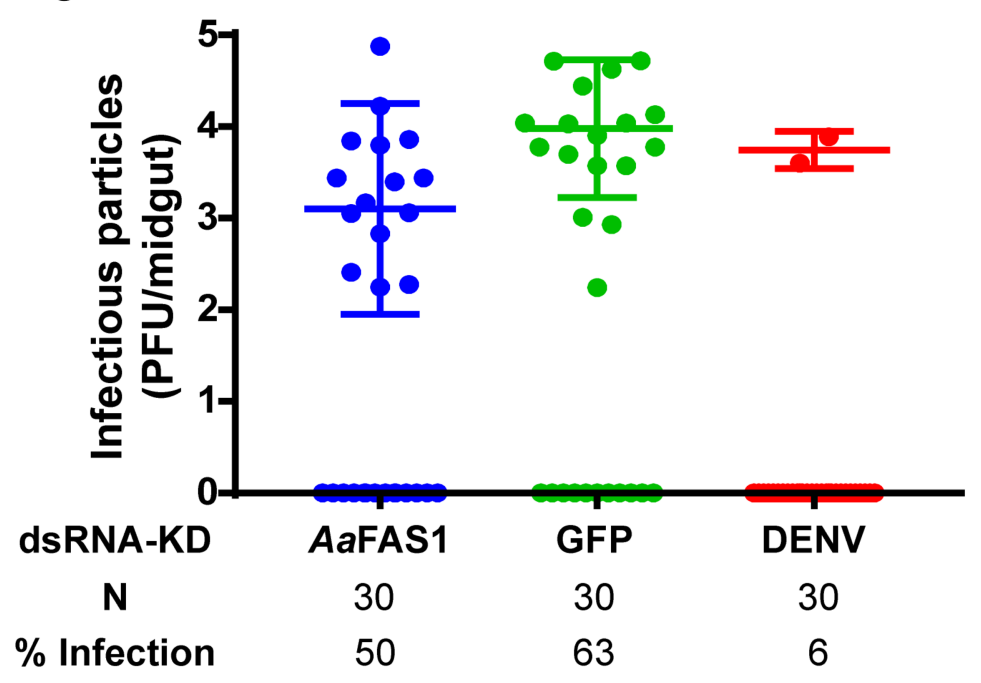

b

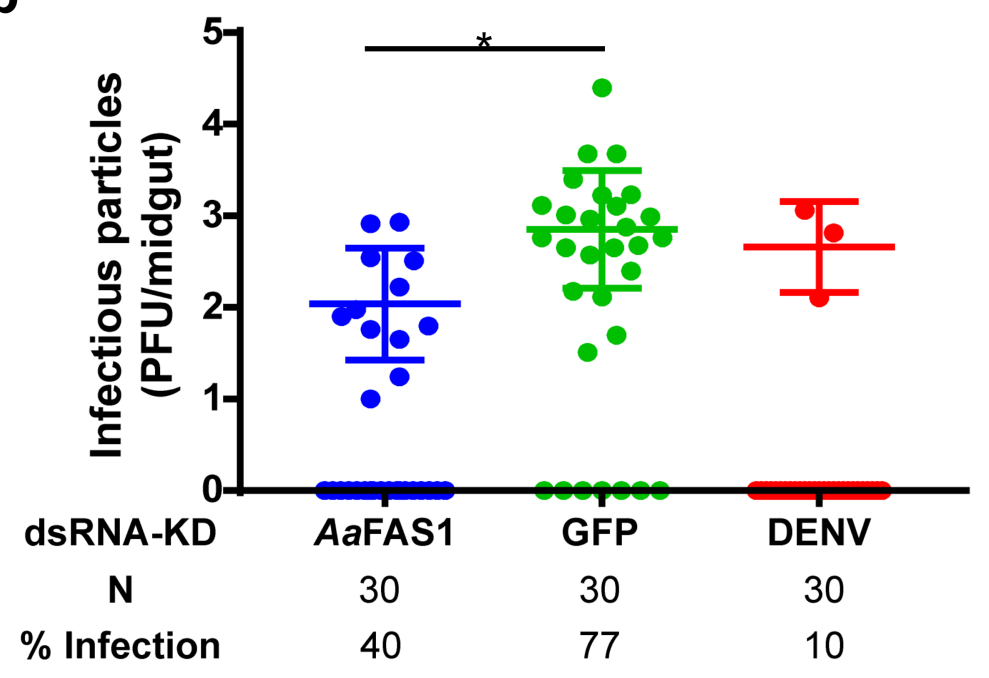

d

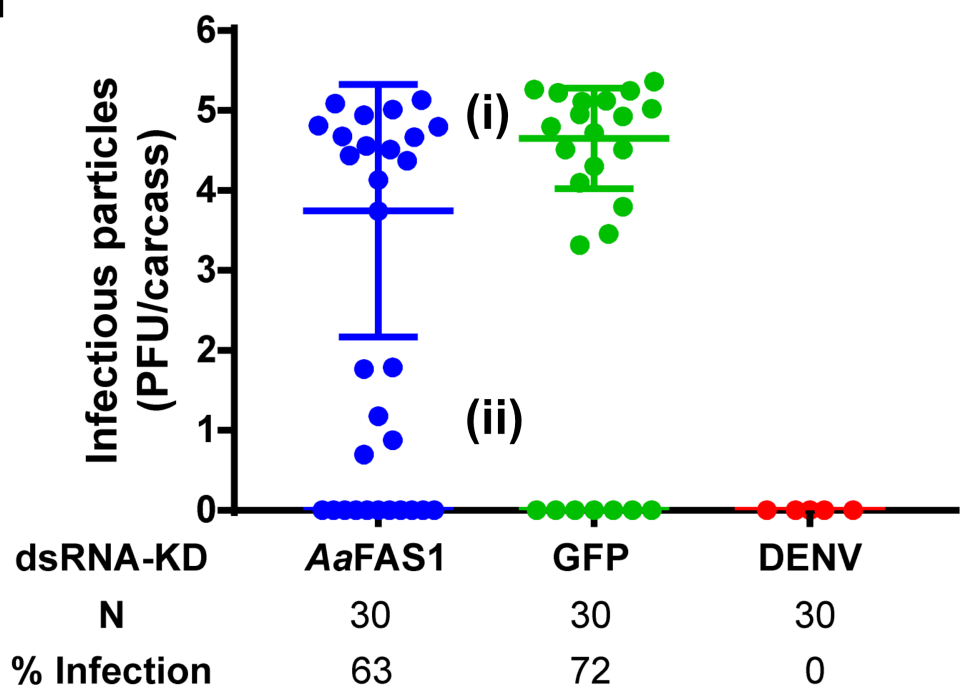

